\title{
Updated checklist of aquatic macrophytes from Northern Brazil
}

\author{
Edson Gomes de MOURA JÚNIOR ${ }^{*}$, Raíssa Maria Sampaio de PAIVA², Angélica Cândida FERREIRA³, \\ Lucília Dias PACOPAHYBA ${ }^{2}$, Aldaléa Sprada TAVARES ${ }^{4}$, Fernando Alves FERREIRA ${ }^{5}$, Arnildo POTT5 \\ 1 Universidade Federal do Vale do São Francisco - Campus Ciências Agrárias, Biology Department, Rodovia BR 407, Lote 543 - Projeto de Irrigação Nilo Coelho s/n, Petrolina, \\ Pernambuco, Brazil. \\ 2 Universidade Federal de Roraima, Biology Department, Av. Capitão Ene Garcez 2413, Boa Vista, Roraima, Brazil. \\ 3 Universidade Federal Rural de Pernambuco, Biology Department, Rua Dom Manoel de Medeiros s/n, Recife, Pernambuco, Brazil. \\ ${ }^{4}$ Universidade Federal de Santa Catarina - Campus Universitário Trindade, Botany Department, Rua Roberto Sampaio Gonzaga s/n, Florianópolis, Santa Catarina, Brazil. \\ ${ }^{5}$ Universidade Federal de Mato Grosso do Sul, Graduate Program in Plant Biology, Cidade Universitária s/n, Campo Grande, Mato Grosso do Sul, Brazil. \\ * Corresponding author: jrbio10@hotmail.com
}

\section{ABSTRACT}

Field collection and herbaria data did not allow to quantify the diversity of aquatic plants from Northern Brazil, so we could not detect biogeographic patterns. Therefore, our objectives were to identify and quantify the aquatic macrophytes of North Brazilian states, analyzing herbaria data plataforms (SpeciesLink and Flora do Brasil). The checklist was produced by bibliographic search (articles published between 1980 and 2000), herbaria collections of the platforms SpeciesLink and Flora do Brasil and field expeditions, where we utilized asystematic sampling. We also analyzed the floristic similarity of aquatic macrophytes among Northern Brazil, wetlands of distinct Brazilian regions and the Neotropics. We recorded 539 species, of which 48 are endemic to Brazil. The states with highest number of species were Amazonas and Pará, independently on platform. The most represented families were Poaceae (89 species), Podostemaceae (55), Cyperaceae (50) and Fabaceae (47). We highlight the unprecedent richness of Podostemaceae, due to our own field collection efforts on favorable habitats, 25 species being endemic. Emergent and/or amphibious plants (515) were dominant in total species richness and were best represented in lotic habitats. We found significant differences in richness and floristics among states, obtained from the platforms. There is floristic similarity among Northern states and other Brazilian wetlands. In conclusion, we observed a rich aquatic flora in Northern Brazil, in spite of scarcity of records for Acre, Rondonia and Tocantins; we highlight the unprecedent number of endemic species of Podostemaceae (25) and contrasting richness between SpeciesLink and Flora do Brasil.

KEYWORDS: floristics, life forms, Podostemaceae, species richness

\section{Lista atualizada de macrófitas aquáticas da região Norte do Brasil}

\section{RESUMO}

Os eforços de campo e os dados de herbários não nos permitiam quantificar a diversidade plantas aquáticas no Norte do Brasil e, com isso, nos impediam de traçar padrôres biogeográficos sobre essa comunidade. Assim, o presente estudo teve por objetivos identificar e quantificar as macrófitas aquáticas dos estados do Norte do Brasil, analisando duas plataformas de dados de herbarios (SpeciesLink e Flora do Brasil). O checklist baseou-se em levantamento bibliográfico (revistas e artigos publicados entre 1980 e 2000) e em coletas de campo, na qual foi utilizada amostragem de "caminhamento". Após a elaboração do checklist, verificaram-se os registros das espécies para os estados da região, consultando-se as coleçóes das plataformas SpeciesLink e Flora do Brasil. Foi analisada a similaridade de especies de macrófitas aquáticas do Neotrópico, Norte do Brasil e ecossistemas aquáticos de distintas regióes do país. Foram registradas 539 espécies, sendo 48 endêmicas do Brasil. As famílias mais representativas foram Poaceae (89 espécies), Podostemaceae (55), Cyperaceae (50) e Fabaceae (47). Ressaltamos a inédita riqueza de Podostemaceae, devida a nossos esforços de coleta em ambientes propícios, 25 espécies sendo endêmicas. Os estados que apresentaram maior número de registros de espécies foram Amazônia e Pará, independentemente da plataforma de dados analisada. As espécies anfíbias e/ou emergentes (505 espécies) foram dominantes na riqueza total dos sete estados analisados. Foram constatadas diferenças significativas quanto aos resultados de riqueza e composiçáo florística dos estados, obtidos pelas plataformas Flora do Brasil e SpeciesLink. A análise de similaridade florística evidenciou a formação de grados entre estados do Norte ou ecossistemas aquáticos brasileiros cujos sistemas climatológicos apresentam semelhanças. Em síntese, observamos uma flora rica de plantas aquáticas no Norte do Brasil, apesar da carência de registros para o Acre, Rondonia e Tocantins. Destacamos o número recorde de espécies endêmicas de Podostemaceae (25 spp.) e as divergências quanto aos resultados de riqueza registradas pelo SpeciesLink e Flora do Brasil.

PALAVRAS-CHAVE: florística, formas de vida, Podostemaceae, riqueza 


\section{INTRODUCTION}

The water surface of the Northern region represents 70\% of the area of Brazil ( $c a .4$ million $\mathrm{km}^{2}$ ), that includes rivers, streams, waterfalls, floodplains, wet savannas, swamps, lakes and reservoirs (Bicudo et al. 2010). This region has the largest hydrographic basin on Earth (Amazonas), which peculiar hydrologic features (white waters, black waters and clear waters) and vegetation types (savannas, floodable forests, white sand savannas or campinalcampinaranas, flood free forests) make Northern Brazil rich in endemism of aquatic biodiversity (Piedade et al. 2010).

Wide latitudinal and longitudinal gradients influence the climatological and limnological systems of micro-basins (Raven et al. 2007). So, the distribution of aquatic species and, consequently, the patterns of richness and composition can vary within Northern Brazil and/or between this region and other Brazilian wetlands.

The Northern region of Brazil is still little known considering species richness and life forms of aquatic macrophytes. According to the species list of Flora do Brasil (Lista de espécies da flora do Brasil 2012), the richness of aquatic plants of this region is estimated as 137 species of strictly aquatic habitat, while the Northeast and the Pantanal exhibit, respectively, 412 and 273 species of aquatic macrophytes and amphibious plants (Pott and Pott 2000; Moura-Júnior et al. 2013). However, the data of SpeciesLink (SPLink 2012) reveal that the richness of aquatic macrophytes for Northern Brazil is around 250 species, with ca. 2500 records in Brazilian herbaria.

According to Moura-Júnior et al. (2013), checklists elaborated after compilation of field data, bibliographic survey, herbaria collection and/or platforms of virtual data (e.g. Flora do Brasil and the project SpeciesLink) allow to increase knowledge on biodiversity in a given study area as well as to identify efficient tools to obtain data. This way, when utilizing Flora do Brasil and SpeciesLink platforms to compile the checklist, we can test whether patterns of species richness and composition of aquatic plants are influenced by the chosen data set. We used both because they are the main databanks on flora in Brazil.

The objective of this work was to: (i) inventory the aquatic macrophyte flora for Northern Brazil, pointing out the endemic species; (ii) determine richness of macrophytes regarding life forms; (iii) determine species richness and floristic patterns among Northern states and other Brazilian regions; and (iv) compare the data platforms Flora do Brasil and SpeciesLink.

\section{MATERIALS AND METHODS}

\section{Description of the study area}

The Northern region has seven states: Acre (AC), Amapá (AP), Amazonas (AM), Pará (PA), Rondônia (RO), Roraima
(RR) and Tocantins (TO) (IBGE 2011) (Figure 1). The climate exhibits spatial variation, being equatorial (predominant) or tropical, with a relatively dry period in June-November, and a rainy season with heavy rainfall, from December to May (monthly mean of $300 \mathrm{~mm}$ ) (INMET 2013). The mean annual rainfall of 2,000 $\mathrm{mm}$, reaching over 3,000 $\mathrm{mm}$ in some areas, such as the littoral of AP, the mouth of the Amazonas river and parts of Western Amazon (INMET 2013). Above the equatorial line, the seasons become inverted in relation to the southern side.

The Northern region has two main watersheds: of the Amazon River and of the Tocantins River (ANA 2012). The Amazon basin is the largest in the world, covering 3.87 million $\mathrm{km}^{2}$ (nearly half Brazil), draining all Northern states (ANA 2012). The Tocantins basin covers 1 million $\mathrm{km}^{2}$ in TO, GO, MT, PA and MA, the main rivers being Araguaia, Tocantins and Itacaiúnas (ANA 2012).
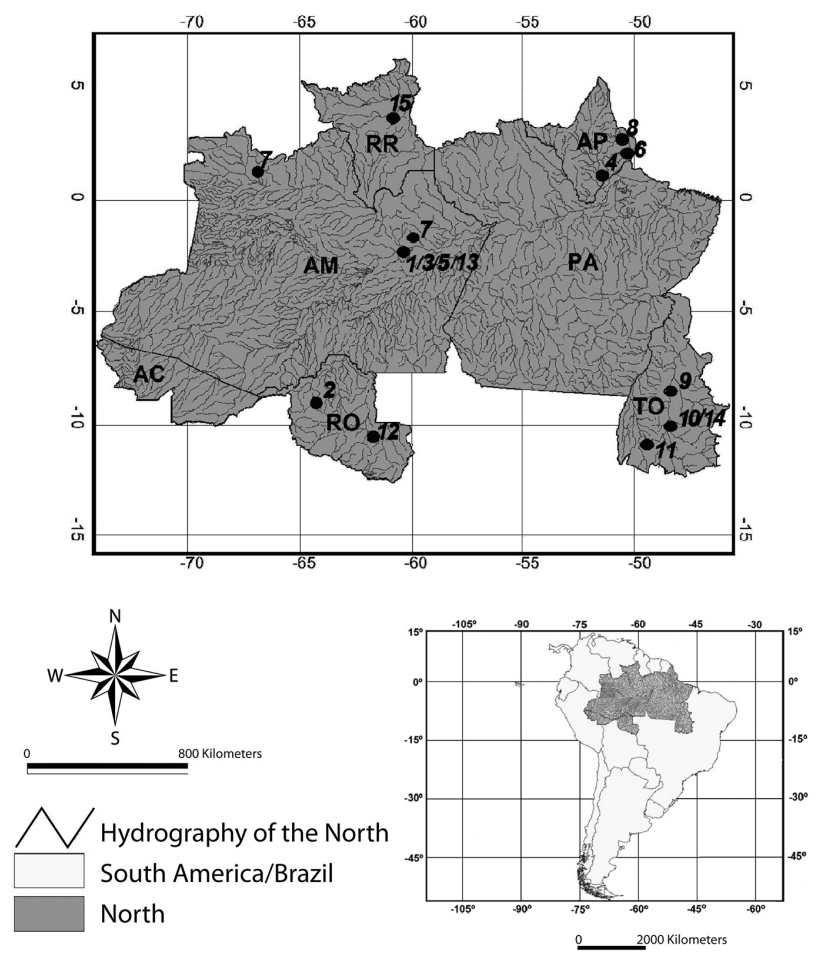

Figure 1. Geographic localization and hydrological map of the Northern region of Brazil. Legend: (1-15) study areas of the consulted sources for compilation of the checklist (Table 1). Northern states: $A C=$ Acre; $A M=$ Amazonas; $A P$ $=$ Amapá; $\mathrm{RO}=$ Rondônia; $\mathrm{RR}=$ Roraima; $\mathrm{PA}=$ Pará; $\mathrm{TO}=$ Tocantins.

\section{Data collection}

We constructed a list of aquatic macrophytes for Northern Brazil. This checklist was generated by a bibliographic search of studies published between August 1980 and 2013. We consulted floristic and/or taxonomic works such as book 
chapters or national and international scientific articles (Table 1). We incorporated many additional species from the author's own field collections to the checklist. Some taxa recorded in the field were identified to the taxonomic level of variety by the specialist author.

The species were collected by authors for over seven years (1994-2001) of field research, in sub-basins of the River Amazonas, Tapajós and Xingu of the different seasons of the hydrological cycle, and specimens are kept in the Herbarium INPA of the Instituto Nacional de Pesquisas da Amazônia, including types of various species. Samplings were performed following the method of fast sampling, similar to the asystematic sampling (Filgueiras et al. 1994). Accordig to Ratter et al. (2003), the technique is based on surveys called wide patrolling, doing at least three walks in strait line in the vegetation and listing the not yet recorded species observed at regular time intervals (5 to 15 minutes).

After compiling the checklist, we verified the species records for the Northern states, consulting the floristic collections of the system SpeciesLink (SPLink 2014) and the species list of Flora do Brasil (Lista de espécies da Flora

Table 1. Consulted studies for the compilation of the checklist of aquatic macrophytes of Northern Brazil with information of the states and respective types of studied habitats. Legend: $\left.{ }^{(1-15}\right)$ - geographic localization of the study area on the map of the Northern region (Figure 1); $\left(^{*}\right)$ - report which sampling area covers the whole Northern region.

\begin{tabular}{|c|c|c|}
\hline References & Studied region & Type(s) of studied habitats \\
\hline Junk and Furch (1980) & $\mathrm{AM}^{1} / \mathrm{RO}^{2}$ & Rivers and anabranches \\
\hline Albuquerque (1981) & North* & $\begin{array}{c}\text { Rivers, floodplains, lakes, } \\
\text { temporary lakes, oxbow } \\
\text { lakes, permanent lakes, } \\
\text { swamps, reservoirs }\end{array}$ \\
\hline Junk and Piedade (1993) & $\mathrm{AM}^{3}$ & Floodplains \\
\hline Costa-Neto et al. (2003) & $\mathrm{AP}^{4}$ & Anabranches \\
\hline Piedade et al. (2005) & $\mathrm{AM}^{5}$ & $\begin{array}{c}\text { Rivers, floodplains, lakes, } \\
\text { temporary lakes, oxbow, } \\
\text { permanent lakes, swamps, } \\
\text { reservoirs }\end{array}$ \\
\hline Tavares et al. (2006) & $\mathrm{AP}^{6} / \mathrm{AM}^{7}$ & Rivers \\
\hline Costa-Neto et al. (2007) & $\mathrm{AP}^{8}$ & Natural lakes \\
\hline Trevelin et al. (2007) & $\mathrm{AM}^{13}$ & Rivers \\
\hline Piedade et al. (2010) & North ${ }^{*}$ & $\begin{array}{l}\text { Rivers, floodplains, lakes, } \\
\text { temporary lakes, oxbow } \\
\text { lakes, permanent lakes, } \\
\text { swamps, reservoirs }\end{array}$ \\
\hline Bianchini Jr. et al. (2010) & $\mathrm{TO}^{9}$ & Reservoirs \\
\hline Lolis and Thomaz (2011) & $\mathrm{TO}^{10}$ & Reservoirs \\
\hline Oliveira et al. (2011) & $\mathrm{TO}^{11}$ & Rivers \\
\hline Souza and Nunes (2011) & $\mathrm{R} 0^{12}$ & Rivers \\
\hline Pinheiro and Lolis (2012) & $\mathrm{TO}^{14}$ & Reservoirs \\
\hline Pinheiro et al. (2012) & $\mathrm{RR}^{15}$ & Natural lakes \\
\hline
\end{tabular}

do Brasil 2014). Afterwards, the results obtained from these data banks were compared regarding richness and species composition. We point out that some species cited in the consulted bibliography for the checklist were so far unknown for Brazil (14 species) or for the Northern region (60 species) (sensu Lista de espécies da Flora do Brasil 2014). The consulted sources did not mention the herbarium record number or collector number of 74 species, impairing their taxonomic checking, therefore they were disregarded.

The species list followed the classification of families proposed by APG III (2009) for angiosperms, by Smith et al. (2006) for pteridophytes, and Buck and Goffinet (2000) for bryophytes. Plant names and respective authors were verified at the data bank of Missouri Botanical Garden (Tropicos 2014), correcting synonyms and invalid names. The concept of aquatic macrophytes followed Fasset (1940): plants visible with the naked eye that occur either permanently or for several months emerse, submerged or floating in fresh water.

Species were classified regarding life forms and types of ecosystems where they were recorded (lentic, lotic or reservoirs). Life forms of macrophytes followed Irgang and Gastal (1996), based on horizontal zoning of species in the ecosystem and on water depth: amphibious - species which colonize the interface between aquatic and terrestrial habitats; epiphytes - plants rooted on organic substrates (emergent and/or floating macrophytes); emergent - plants rooted on the bottom, emerse leaves and flowers, occurring in shallow areas and close to the shore; submerged and floating - species of deep and central zones of the water body occurring under or on top of the water column, respectively.

The species were assorted into life forms following information given by the consulted articles. The species cited in articles without mentioning life forms were classified according to specific literature (Pott and Pott 2000; MouraJunior et al. 2013). Some species show more than one life form, e.g. emergent/amphibious or emergent/epiphyte.

The types of habitats were categorized as: lotic - rivers or streams; lentic - lakes and ponds; and intermediate ones - reservoirs or ecosystems with abiotic characteristics similar to either lotic or lentic habitats (Thornton 1990; Esteves 2011). The species were categorized into habitats following information given by the consulted articles.

\section{Analyses of data}

For the objectives (i), (ii) and (iii) we used descriptive statistics. We ran cluster analyses to evaluate the floristic similarity of aquatic plants among the Northern states (gathered from Flora do Brasil and SpeciesLink) or between the Northern region and other Brazilan large wetlands. Floristic similarity was considered as percentage of species composition coincident between two environments. The 
matrices of flora were submitted to analyses of similarity using Jaccard index (Magurran 2004) and ordinated by WPGMA, applying the software PRIMER pc. 6.0 (Clarke and Gorley 2006). The index of Jaccard $(\mathrm{Sj})$ was utilized to compare the floristic composition of the states from different plataforms of analyzed data, as well as to evaluate the floristic similarity of macrophytes of the North with the Neotropics and other Brazilian wetlands.

$$
S j=a /(a+b+c)
$$

where " $a$ " is the number of species found in both sites A and $\mathrm{B}$, "b" is the number of species in site B, but not in A, "c" is the number of species in site A, but not in B.

To test consistency of clusters we utilized the method of Monte Carlo with 1000 replicates and $\alpha=5 \%$, using the program RandMat version 1.0 (Manly 1997). Based on available records we calculated the percentage of species occurrence, according to Mateucci and Colma (1982).

We used the the software StimateS 9.10 (Colwell 2013) for expected richness of aquatic macrophytes of Northern Brazil and their minimum and maximum confidence intervals (95\% CI), according to Flora do Brasil and SpeciesLink. The expected richness values and the CIs for each platform were calculated after the floristic matrix of the states (sampling units), that was later rarefacted to 50 samples (stabilization point for expected richness). The rarefaction curve for expected richness was elaborated after the formula established by Sanders (1968) and modified by Simberloff (1972):

$$
\mathrm{E}\left(\mathrm{S}_{\mathrm{n}}\right)=\sum_{i=1}^{\mathrm{S}}\left[1-\frac{N / N_{i}}{n} /\left(\frac{N}{n}\right)\right]
$$

where $\mathrm{E}(\mathrm{S})$ is the expected number of species in a random sampling, $\mathrm{S}$ is the total number of recorded species, $N$ os the total number of recorded individuals, $N i$ is the number of individuals of species $i$, and $n$ is the standardized size of the chosen sample. To test significance of differences between the richness obtained from Flora do Brasil and SpeciesLink, we compared the CIs of these platforms.

\section{RESULTS}

Based on bibliographic search and field expeditions we identified 539 species of aquatic macrophytes for Northern Brazil, of which 48 species are endemic to Brazil, standing out 25 Podostemaceae (Table 2).

Data from Flora do Brasil and SpeciesLink evidenced that the states AM and PA had the highest species richness. The results obtained from Flora do Brasil showed respectively 410 and 434 species for AM and PA, while SpeciesLink contemplated 430 and 432 species, respectively (Table 2 ). The minimum richness CI considering Flora do Brasil was 558 species, and the estimated maximum according to SpeciesLink was 551 species (Figure 2). Upon the estimated CIs, we verified significant differences regarding richness between Flora do Brasil and SpeciesLink (Figure 2).

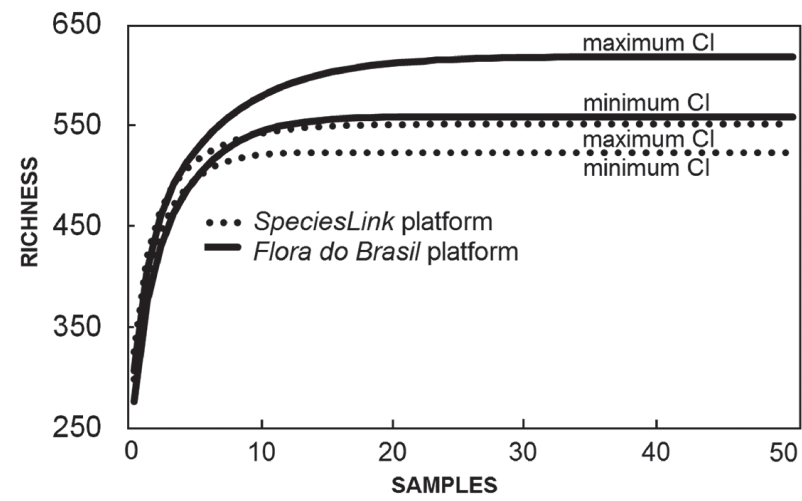

Figure 2. Rarefaction curve for expected richness of aquatic macrophytes of Northern Brazil, based on data of the platforms SpeciesLink and Flora do Brasil. Legend: (Line and dotted) - confidence interval maximum and minimum $(\mathrm{Cl}$ $95 \%$ ) of the expected richness, extrapolated for 50 sampling units.

The families which most contributed to total richness of aquatic macrophytes in Northern Brazil were Poaceae (89 species), Podostemaceae (55), Cyperaceae (50) and Fabaceae (47), that added to over $40 \%$ of the recorded species in each state (Figure 2). Other families with considerable richness of aquatic macrophytes in this region were Convolvulaceae (16), Malvaceae (16), Asteraceae (15), Polygalaceae (14), Lamiaceae (12), Onagraceae (12), Lentibulariaceae (11) and Rubiaceae (10), that altogether contributed to over $15 \%$ of the aquatic flora of the studied states (Table 2).

From the total inventoried macrophytes, 52 species $(9.6 \%)$ presented records for all Northern states, independently on the platform. Among plants spread over the entire region, Cyperus haspan L., C. luzulae (L.) Retz. and Eleocharis interstincta (Vahl) Roem. \& Schult. occurred in all habitats, what explains their presence in the whole study area (Table 2).

According to the Monte Carlo test, the states with similarity of Jaccard scores $(\mathrm{J})>0.50$ (or $50 \%$ ) were considered significantly $(\mathrm{p}<0.05)$ similar in floristic composition of aquatic macrophytes. Within this significance limit we did not observe differences regarding floristic data of SpeciesLink and Flora do Brasil, except for TO (Figure 3A). After data extraction from the platforms we observed the formation of two grades, following a latitudinal gradient: $1^{\text {st }}$ - states of AP, AM, PA and RR (latitude between 4 and $-9^{\circ}$ ); and $2^{\text {rd }}-$ AC and RO (latitude between -6 and $-12^{\circ}$ ) (Figures $1,3 \mathrm{~A}$ ). Yet, TO did not show floristic similarity with other Northern states, independently on platform (Figures 1, 3A). 
Through analysis of floristic similarity of aquatic macrophytes among the Neotropics, the Northern region and wetlands of distinct Brazilian regions, we also found a latitudinal gradient, with formation of three grades $(J=0.12)$ : the first formed by North and Northeast; the second composed of the Pantanal wetland and the Paraná river floodplain; and the third represented by wetlands of Rio Grande do Sul (Figure 3B). However, the formation of these grades was not confirmed by the Monte Carlo test, which limit of significance was Jaccard J $>0.40(40 \%)$ (Figure 3B). The results of cluster analysis also evidenced low floristic similarity $(J=0.04)$ of aquatic macrophytes between Northern Brazil and the world checklist, considering that out of 539 species recorded for this region, $329(75 \%)$ had not yet been incorporated to the checklist of the Neotropics.
The species with amphibious and/or emergent life forms were dominant in the flora of aquatic macrophytes of Northern Brazil (498 species), together representing over 90\% of the species richness. The states AM and PA contemplated 13 categories of life forms each, independently on the platform (Table 2). According to the data extracted from SpeciesLink, AC, AP, RO, RR and TO exhibited 11, 12, 11, 12 and 12 life forms, respectively, while for Flora do Brasil we counted 12 (AC, AP, RR), 11 (TO) and 9 forms (RO).

Lentic habitats of Northern Brazil showed higher richness of aquatic macrophytes (517 species), more representative when compared to lotic (293 species) and transition ecosystems (38 species), the states AP, AM, PA and RR being the most influent in such results (Table 2).
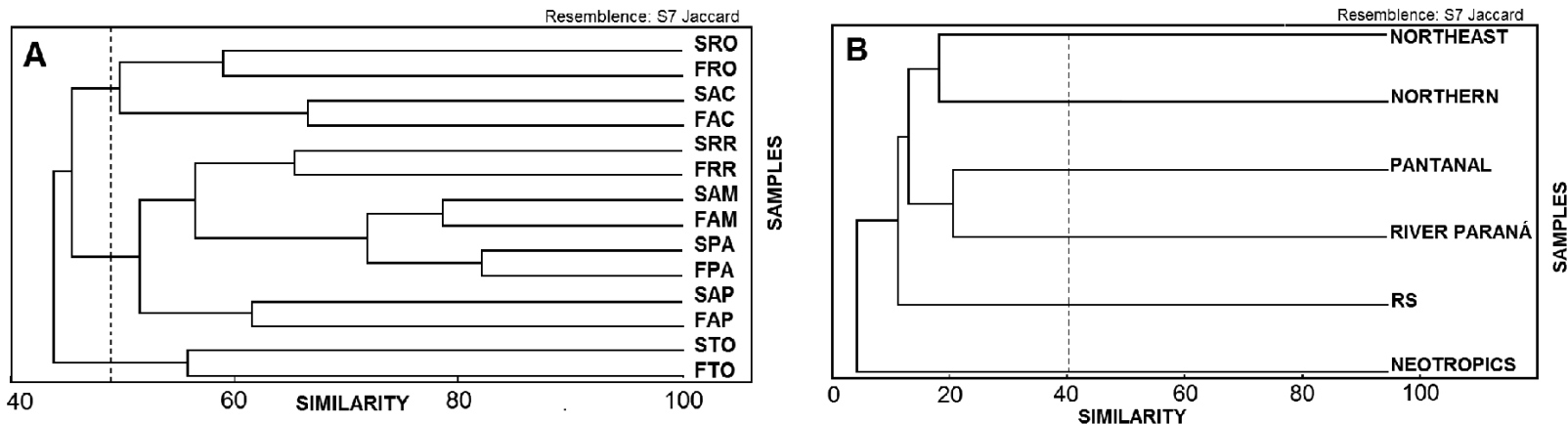

Figure 3. Floristic similarity (index of Jaccard) of aquatic macrophytes of the Northern states of Brazil (A) or of North with the Neotropics and aquatic ecosystems of distinct regions of Brazil (B), and Monte Carlo permutation test (with 1000 replications, $\alpha=5 \%$ ). Legends: (SAC) SpeciesLink - Acre; (SAP) SpeciesLink - Amapá; (SAM) SpeciesLink - AM; (SPA) SpeciesLink - Pará; (SRO) SpeciesLink - Rondônia; (SRR) SpeciesLink - Roraima; (STO) SpeciesLink - Tocantins; (FAC) Flora do Brasil - Acre; (FAP) Flora do Brasil - Amapá; (FAM) Flora do Brasil - Amazonas; (FPA) Flora do Brasil - Pará; (FRO) Flora do Brasil - Rondônia; (FRR) Flora do Brasil - Roraima; (FTO) Flora do Brasil - Tocantins.

Table 2. List of families, species and life forms (LF) of aquatic macrophytes in the states of Northern Brazil, with information on endemism (END), life form (LF), and type of ecosystem/habitat with record (HAB). Legend: (F) record on platform Flora do Brasil; (S) record on platform SpeciesLink; (A) Amphibious; (EM) Emergent; (FF) Free floating; (RF) Rooted floating; (RS) Rooted submersed; (FS) Free submersed; (EP) Epiphyte; (LEN) Lentic ecosystem; (TRA) Transition ecosystem; (LOT) Lotic ecosystem; (+) presence of information; (-) absence of information; (*) species not in checklist of Neotropics; $\left(^{*}\right)$ species recorded in the field; (a) lotic habitats; (b) reservoirs; (c) lentic habitats; (d) species recorded in three types of habitats.

\begin{tabular}{|c|c|c|c|c|c|c|c|c|c|c|c|c|c|c|c|c|c|}
\hline \multirow{2}{*}{ Family/ Species } & \multirow{2}{*}{ LF } & \multicolumn{2}{|c|}{ AC } & \multicolumn{2}{|c|}{$\mathrm{AP}$} & \multicolumn{2}{|c|}{ AM } & \multicolumn{2}{|c|}{ PA } & \multicolumn{2}{|c|}{ Ro } & \multicolumn{2}{|c|}{ RR } & \multicolumn{2}{|c|}{ TO } & \multirow{2}{*}{ END } & \multirow{2}{*}{$\mathrm{HAB}$} \\
\hline & & $\mathrm{F}$ & $S$ & $\mathrm{~F}$ & S & $\mathrm{F}$ & $S$ & $\mathrm{~F}$ & S & $\mathrm{F}$ & S & $\mathrm{F}$ & S & $\mathrm{F}$ & $S$ & & \\
\hline \multicolumn{18}{|l|}{ Acanthaceae } \\
\hline *Justicia comata (L.) Lam. & EM & + & + & - & + & + & + & + & + & + & + & - & - & - & - & - & $c$ \\
\hline *Justicia laevilinguis (Nees) Lindau & EM & - & - & - & - & + & + & + & + & + & + & - & - & - & - & - & c \\
\hline *Justicia pectoralis Jacq. & A & + & - & - & - & + & + & + & - & + & - & + & - & - & - & - & c \\
\hline \multicolumn{18}{|l|}{ Alismataceae } \\
\hline \#Echinodorus grisebachii Small & EM & - & - & + & + & + & + & + & - & + & + & - & + & - & - & - & $\mathrm{a}$ \\
\hline Echinodorus Iongipetalus Micheli & EM & - & - & - & - & - & - & - & - & - & - & - & - & + & + & - & $d$ \\
\hline Echinodorus macrophyllus (Kunth) Micheli & EM & - & - & - & + & - & - & - & - & + & + & + & + & - & - & - & $\mathrm{a}, \mathrm{c}$ \\
\hline Echinodorus paniculatus Micheli & EM & - & - & + & + & - & - & + & + & - & - & - & + & - & - & + & $b$ \\
\hline Echinodorus scaber Rataj & EM & - & - & - & - & + & + & + & + & - & - & - & - & - & - & - & c \\
\hline Helanthium tenellum (Mart.) Britton & $\mathrm{EM} / \mathrm{A}$ & - & - & - & - & - & + & + & + & + & + & + & + & - & + & - & $d$ \\
\hline Hydrocleys nymphoides (Willd.) Buchenau & RF & - & + & - & - & - & + & + & + & - & - & - & - & - & - & - & $\mathrm{a}, \mathrm{c}$ \\
\hline
\end{tabular}


Table 2. (Continuation)

\begin{tabular}{|c|c|c|c|c|c|c|c|c|c|c|c|c|c|c|c|c|c|}
\hline \multirow{2}{*}{ Family/ Species } & \multirow{2}{*}{ LF } & \multicolumn{2}{|c|}{$A C$} & \multicolumn{2}{|c|}{ AP } & \multicolumn{2}{|c|}{ AM } & \multicolumn{2}{|c|}{ PA } & \multicolumn{2}{|c|}{ Ro } & \multicolumn{2}{|c|}{$\mathrm{RR}$} & \multicolumn{2}{|c|}{ TO } & \multirow{2}{*}{ END } & \multirow{2}{*}{$\mathrm{HAB}$} \\
\hline & & $\mathrm{F}$ & $\mathrm{S}$ & $\mathrm{F}$ & $S$ & $\mathrm{~F}$ & $S$ & $\mathrm{~F}$ & $\mathrm{~S}$ & $\mathrm{~F}$ & $S$ & $\mathrm{~F}$ & $S$ & $\mathrm{~F}$ & S & & \\
\hline Limnocharis flava (L.) Buchenau & $E M / A$ & + & + & + & + & + & + & + & + & - & + & + & + & + & + & - & $d$ \\
\hline Sagittaria guayanensis Kunth & $\mathrm{EM} / \mathrm{FF}$ & + & + & - & - & + & + & + & + & - & + & + & + & + & + & - & $d$ \\
\hline Sagittaria rhombifolia Cham. & EM & - & - & - & + & - & - & + & + & - & - & + & + & + & + & - & c \\
\hline Sagittaria sprucei Micheli & EM & + & + & - & - & + & + & + & + & - & - & - & - & - & - & - & c \\
\hline \multicolumn{18}{|l|}{ Amaranthaceae } \\
\hline *Alternanthera brasiliana (L.) Kuntze & A & - & - & - & - & - & - & + & + & + & + & - & - & + & - & - & $c$ \\
\hline *Alternanthera paronychioides A. St.-Hil. & A & - & - & - & - & + & + & + & + & - & - & - & - & - & - & - & $a, c$ \\
\hline *Alternanthera pilosa Moq. & A & + & + & - & - & + & + & + & + & - & + & + & + & - & - & - & $a, c$ \\
\hline Alternanthera tenella Colla & A & + & + & - & - & + & + & + & + & + & + & + & + & - & - & - & $\mathrm{C}$ \\
\hline *Amaranthus spinosus L. & A & + & + & - & - & + & + & - & + & - & - & - & + & + & + & - & c \\
\hline *Amaranthus viridis $\mathrm{L}$. & A & - & - & - & - & + & + & + & + & - & - & - & - & - & - & - & $a, c$ \\
\hline${ }^{*}$ Chamissoa altissima (Jacq.) Kunth & A & + & + & - & - & + & + & - & + & - & + & - & + & - & - & - & c \\
\hline *Chenopodium ambrosioides L. & A & + & + & + & + & + & + & + & + & + & + & + & + & + & + & - & C \\
\hline \multicolumn{18}{|l|}{ Apocynaceae } \\
\hline *Allamanda nobilis T. Moore & EM & - & - & + & - & + & + & - & + & + & - & + & + & - & - & - & $\mathrm{C}$ \\
\hline *Mandevilla hirsuta (A.Rich.) K. Schum. & EM & + & + & + & + & + & + & + & + & + & + & + & + & + & + & - & $\mathrm{C}$ \\
\hline *Odontadenia nitida (Vahl) Müll. Arg. & A & + & + & + & + & + & + & + & + & + & + & + & + & - & + & - & $\mathrm{c}$ \\
\hline *Rhabdadenia biflora (Jacq.) Müll. Arg. & A & - & - & + & + & - & + & + & + & - & + & - & + & - & - & - & c \\
\hline *Rhabdadenia madida (Vell.) Miers & A & - & - & + & + & + & + & + & + & - & + & - & + & - & + & - & $a, c$ \\
\hline \multicolumn{18}{|l|}{ Araceae } \\
\hline *Dracontium longipes Engl. & A & + & + & - & - & + & + & - & - & - & - & - & - & - & - & + & c \\
\hline Lemna aequinoctialis Welw. & $\mathrm{FF}$ & - & - & - & - & + & + & - & - & - & - & - & - & - & - & - & $c$ \\
\hline Lemna valdiviana Phil. & $\mathrm{FF}$ & - & - & - & - & + & + & - & - & - & - & - & - & - & - & - & c \\
\hline Montrichardia arborescens (L.) Schott & A & + & + & - & + & + & + & + & + & + & + & - & + & - & - & - & $a, c$ \\
\hline Montrichardia linifera (Arruda) Schott & EM & - & - & + & + & + & + & + & + & - & - & + & + & - & - & - & c \\
\hline Pistia stratiotes L. & $\mathrm{FF}$ & + & + & + & + & + & + & + & + & - & - & - & + & - & - & - & $d$ \\
\hline Spirodela intermedia W. Koch & $\mathrm{FF}$ & - & - & - & - & - & + & + & - & - & - & - & - & - & - & - & $c$ \\
\hline Urospatha sagittifolia (Rudge) Schott & $\mathrm{EM} / \mathrm{A}$ & + & + & + & + & + & + & + & + & - & + & - & - & + & + & - & $\mathrm{b}, \mathrm{c}$ \\
\hline *Xanthosoma striatipes (Kunth \& Bouché) Madison & EM & + & + & - & - & + & + & + & + & - & + & - & + & + & + & - & $\mathrm{b}$ \\
\hline \multicolumn{18}{|l|}{ Arecaceae } \\
\hline *Bactris maraja Mart. & A & + & + & - & + & + & + & + & + & + & + & + & + & - & + & - & $a, c$ \\
\hline *Mauritia flexuosa L.f. & A & + & + & - & - & + & + & + & + & + & + & - & + & + & + & - & $c$ \\
\hline *Mauritiella armata (Mart.) Burret & A & + & - & - & - & + & + & + & + & + & + & + & + & + & + & - & $c$ \\
\hline \multicolumn{18}{|l|}{ Asteraceae } \\
\hline *Ambrosia artemisiaefolia L. & A & + & + & + & + & + & + & + & + & + & + & - & - & - & - & - & c \\
\hline${ }^{*}$ Conyza bonariensis (L.) Cronquist & A & + & + & + & + & + & + & + & + & + & + & + & + & + & + & - & $c$ \\
\hline *Conyza sumatrensis (Retz.) E.Walker & A & - & - & - & - & - & + & - & - & + & + & - & - & - & - & - & c \\
\hline \#Eclipta prostrata (L.) L. & EM & + & + & - & - & - & + & - & - & - & - & - & - & - & - & - & $d$ \\
\hline *Egletes viscosa (L.) Less. & $\mathrm{EM} / \mathrm{A}$ & + & + & - & - & + & + & + & + & + & + & - & - & + & + & - & $a, c$ \\
\hline *Emilia sonchifolia (L.) DC. ex Wight & A & - & - & - & + & + & + & + & + & - & + & - & + & - & + & - & c \\
\hline *Erechtites hieracifolius (L.) Raf. ex DC. & A & + & + & - & - & + & + & + & + & - & + & - & - & - & + & - & $\mathrm{c}$ \\
\hline Gymnocoronis spilanthoides DC. & A & - & - & - & - & - & + & + & + & - & - & - & - & - & - & - & c \\
\hline *Mikania congesta DC. & A & - & - & - & + & + & + & + & + & + & + & + & + & - & - & - & $\mathrm{c}$ \\
\hline *Mikania cordifolia (L. f.) Willd. & A & - & + & - & + & + & + & - & + & - & + & - & + & - & + & - & c \\
\hline Pacourina edulis Aubl. & A & + & - & + & + & + & + & + & + & - & - & - & - & - & - & - & c \\
\hline *Sphagneticola trilobata (L.) Pruski & A & + & + & + & + & + & + & + & + & + & + & + & - & + & - & - & $\mathrm{C}$ \\
\hline
\end{tabular}


Table 2. (Continuation)

\begin{tabular}{|c|c|c|c|c|c|c|c|c|c|c|c|c|c|c|c|c|c|}
\hline \multirow{2}{*}{ Family/ Species } & \multirow{2}{*}{ LF } & \multicolumn{2}{|c|}{$\mathrm{AC}$} & \multicolumn{2}{|c|}{ AP } & \multicolumn{2}{|c|}{ AM } & \multicolumn{2}{|c|}{$\mathrm{PA}$} & \multicolumn{2}{|c|}{ RO } & \multicolumn{2}{|c|}{$\mathrm{RR}$} & \multicolumn{2}{|c|}{ TO } & \multirow{2}{*}{ END } & \multirow{2}{*}{$\mathrm{HAB}$} \\
\hline & & $\mathrm{F}$ & $S$ & $\mathrm{~F}$ & S & $\mathrm{F}$ & $S$ & $\mathrm{~F}$ & S & $\mathrm{F}$ & S & $\mathrm{F}$ & $S$ & $\mathrm{~F}$ & $S$ & & \\
\hline *Tilesia baccata (L.f.) Pruski & A & + & + & + & + & + & + & + & + & + & + & + & + & + & + & - & c \\
\hline *Trichospira verticillata (L.) S.F.Blake & A & - & - & + & - & + & + & + & + & - & - & + & + & - & - & - & c \\
\hline *Wedelia calycina Rich. & A & + & - & - & - & + & - & + & + & + & - & + & - & - & - & - & c \\
\hline \multicolumn{18}{|l|}{ Bignoniaceae } \\
\hline${ }^{\star}$ Pleonotoma clematis (Kunth) Miers & A & + & - & + & + & + & + & + & + & + & + & + & + & - & - & - & c \\
\hline *Tanaecium pyramidatum (Rich.) L.G.Lohmann & A & + & + & + & + & + & + & + & + & + & - & + & + & + & - & - & c \\
\hline \#Tynanthus polyanthus (Bureau) Sandwith & A & + & + & - & - & + & + & + & + & + & + & - & - & - & - & - & c \\
\hline \multicolumn{18}{|l|}{ Blechnaceae } \\
\hline *Blechnum serrulatum Rich. & $\mathrm{EM} / \mathrm{A}$ & - & - & + & + & + & + & + & + & + & + & + & + & - & + & - & c \\
\hline \multicolumn{18}{|l|}{ Boraginaceae } \\
\hline${ }^{\star}$ Cordia tetrandra Aubl. & A & + & + & + & + & + & + & + & + & + & + & + & + & + & - & - & $c$ \\
\hline${ }^{\#}$ Euploca procumbens (Mill.) Diane \& Hilger & A & + & + & - & - & - & + & + & + & + & + & + & - & + & - & - & c \\
\hline Heliotropium indicum $\mathrm{L}$. & A & + & + & + & + & + & + & - & + & + & + & - & + & - & + & - & $a, c$ \\
\hline \multicolumn{18}{|l|}{ Cabombaceae } \\
\hline Cabomba aquatica Aubl. & RS & - & - & + & - & + & + & + & + & - & - & + & + & - & - & - & $a, c$ \\
\hline Cabomba furcata Schult. \& Schult. f. & RS & - & - & - & - & + & + & + & + & - & - & + & + & - & + & - & $d$ \\
\hline \multicolumn{18}{|l|}{ Cannaceae } \\
\hline Canna glauca L. & EM & - & - & - & - & - & + & + & + & - & - & + & + & - & + & - & c \\
\hline Cleomaceae & & & & & & & & & & & & & & & & & \\
\hline *Tarenaya spinosa (Jacq.) Raf. & A & + & - & - & - & + & - & + & + & + & - & + & + & + & - & - & $a, c$ \\
\hline Commelinaceae & & & & & & & & & & & & & & & & & \\
\hline *Aneilema umbrosum (Vahl) Kunth & A & + & - & - & - & + & + & + & + & - & - & - & - & - & - & - & c \\
\hline${ }^{\star}$ Commelina erecta $\mathrm{L}$. & A & - & - & - & - & + & + & + & + & + & + & - & - & + & + & - & $a, c$ \\
\hline *Dichorisandra hexandra (Aubl.) Kuntze ex Hand.-Mazz. & A & + & + & + & + & + & + & + & + & + & + & + & + & + & + & - & c \\
\hline Convolvulaceae & & & & & & & & & & & & & & & & & \\
\hline *Aniseia martinicensis(Jacq.) Choisy & $\mathrm{A} / \mathrm{EP}$ & + & + & + & + & + & + & + & + & + & + & + & + & + & + & - & $\mathrm{a}, \mathrm{c}$ \\
\hline *Evolvulus nummularius (L.) L. & A & - & - & + & + & + & + & + & + & - & - & - & - & + & + & - & c \\
\hline */pomoea alba L. & A & + & + & + & + & + & + & + & + & + & + & + & - & + & + & - & c \\
\hline Ipomoea aquatica Forssk. & A & - & - & - & - & + & + & + & - & - & - & - & - & - & - & - & c \\
\hline Ipomoea asarifolia (Desr.) Roem. \& Schult. & $\mathrm{EM} / \mathrm{A}$ & + & + & + & + & + & + & + & + & + & + & + & + & - & + & - & $\mathrm{a}, \mathrm{c}$ \\
\hline \#Ipomoea batatas (L.) Poir. & $\mathrm{A} / \mathrm{EP}$ & + & - & + & + & + & + & + & + & - & - & + & + & + & - & - & c \\
\hline Ipomoea carnea Jacq. & $\mathrm{EM} / \mathrm{A}$ & + & + & + & - & + & + & + & + & - & + & + & - & + & + & - & $a, c$ \\
\hline */pomoea cynanchifolia Meisn. & A & - & - & - & - & + & + & + & + & + & - & + & - & - & - & - & c \\
\hline \#Ipomoea philomega (Vell.) House & A & + & + & + & + & + & + & + & + & + & + & + & + & + & + & - & $c$ \\
\hline *Ipomoea quamoclit L. & A & + & + & - & - & + & + & + & + & + & + & - & - & - & + & - & c \\
\hline *Ipomoea setifera Poir. & A & - & + & - & + & + & + & + & + & - & - & - & + & + & + & - & $c$ \\
\hline *Ipomoea squamosa Choisy & A & - & - & + & + & + & + & + & + & + & + & + & + & + & + & - & c \\
\hline "Iseia luxurians (Moric.) O'Donell & A & - & + & - & + & + & + & + & - & + & + & + & + & - & - & - & c \\
\hline *Merremia umbellata (L.) Hallier f. & A & + & + & - & + & + & + & + & + & + & + & + & + & + & + & - & c \\
\hline *Operculina hamiltonii (G.Don) D.F.Austin \& Staples & A & + & + & + & - & + & + & + & + & + & + & + & - & + & + & - & c \\
\hline *Tetralocularia pennellii 0’Donell & A & + & - & - & - & + & + & + & + & - & - & - & - & - & - & - & c \\
\hline Costaceae & & & & & & & & & & & & & & & & & \\
\hline *Costus arabicus L. & A & + & + & + & + & + & + & + & + & + & + & + & + & - & + & - & c \\
\hline Cucurbitaceae & & & & & & & & & & & & & & & & & \\
\hline *Elaterium amazonicum Mart. & $\mathrm{EM} / \mathrm{A}$ & - & - & - & + & + & - & + & + & - & - & - & - & - & - & - & c \\
\hline${ }^{\star}$ Gurania bignoniacea (Poepp. \& Endl.) C.Jeffrey & A & + & + & + & + & + & + & + & + & + & + & + & + & - & - & - & c \\
\hline
\end{tabular}


Table 2. (Continuation)

\begin{tabular}{|c|c|c|c|c|c|c|c|c|c|c|c|c|c|c|c|c|c|}
\hline \multirow{2}{*}{ Family/ Species } & \multirow{2}{*}{ LF } & \multicolumn{2}{|c|}{$\mathrm{AC}$} & \multicolumn{2}{|c|}{ AP } & \multicolumn{2}{|c|}{ AM } & \multicolumn{2}{|c|}{ PA } & \multicolumn{2}{|c|}{ R0 } & \multicolumn{2}{|c|}{$\mathrm{RR}$} & \multicolumn{2}{|c|}{ TO } & \multirow{2}{*}{ END } & \multirow{2}{*}{$\mathrm{HAB}$} \\
\hline & & $\mathrm{F}$ & $S$ & $\mathrm{~F}$ & $S$ & $\mathrm{~F}$ & $S$ & $\mathrm{~F}$ & $S$ & $\mathrm{~F}$ & $S$ & $\mathrm{~F}$ & $S$ & $\mathrm{~F}$ & $S$ & & \\
\hline *Gurania tricuspidata Cogn. & A & - & - & - & - & + & + & - & - & + & + & - & - & - & - & - & c \\
\hline *Luffa operculata (L.) Cogn. & A & - & - & - & - & + & + & - & + & - & - & - & - & - & - & - & c \\
\hline *Momordica charantia L. & A & + & + & + & + & - & + & - & + & - & + & - & + & + & + & - & c \\
\hline \multicolumn{18}{|l|}{ Cyperaceae } \\
\hline${ }^{\star}$ Abildgaardia ovata (Burm. f.) Kral & A & + & - & - & - & + & - & + & + & + & - & + & + & - & - & - & c \\
\hline *Becquerelia cymosa Brongn. & A & + & + & + & - & + & + & + & + & + & + & + & + & + & - & - & c \\
\hline${ }^{\star}$ Bulbostylis capillaris (L.) C.B. Clarke & A & + & - & + & + & + & + & + & + & + & - & + & + & + & - & - & c \\
\hline *Bulbostylis juncoides (Vahl) Kük. & A & + & - & + & - & + & + & + & + & + & - & + & - & + & + & - & c \\
\hline *Bulbostylis truncata (Nees) M.T. Strong & $A$ & - & - & + & - & + & + & + & + & - & - & + & - & + & + & - & c \\
\hline Cyperus aggregatus (Willd.) Endl. & $A$ & + & + & + & + & + & + & + & + & + & + & + & + & + & + & - & $a, c$ \\
\hline Cyperus articulatus L. & A & - & - & - & + & + & + & + & + & - & - & - & - & - & - & + & $\mathrm{a}, \mathrm{c}$ \\
\hline Cyperus chalaranthus J.PresI \& C.Presl & A & + & + & - & - & - & + & - & - & - & - & - & - & - & + & - & c \\
\hline${ }^{\star}$ Cyperus compressus L. & A & + & - & + & + & + & + & + & + & + & - & + & + & + & + & - & $a, c$ \\
\hline Cyperus distans $\mathrm{L}$. & A & + & - & + & + & + & + & + & + & + & - & + & + & + & - & - & $a, c$ \\
\hline${ }^{\star}$ Cyperus esculentus L. & A & + & + & + & + & + & + & + & + & + & + & + & + & + & + & - & $a, c$ \\
\hline Cyperus giganteus Vahl & A & + & - & + & + & + & + & + & + & + & + & + & + & + & + & - & $a, b$ \\
\hline *Cyperus haspan L. & A & + & + & + & + & + & + & + & + & + & + & + & + & + & + & - & $d$ \\
\hline Cyperus ligularis L. & A & - & + & + & + & + & + & + & + & - & - & + & + & + & - & - & c \\
\hline Cyperus Iuzulae (L.) Retz. & A & + & + & + & + & + & + & + & + & + & + & + & + & + & + & - & $d$ \\
\hline Cyperus odoratus L. & A & + & + & + & + & + & + & + & + & + & + & + & + & + & + & + & $a, c$ \\
\hline${ }^{\star}$ Cyperus rigens C.Presl & A & - & - & - & - & - & + & - & + & - & - & - & - & + & - & - & $a, c$ \\
\hline *Cyperus rotundus L. & A & + & - & + & + & + & + & + & + & + & + & + & + & + & + & + & c \\
\hline Cyperus surinamensis Rottb. & $\mathrm{EM} / \mathrm{A}$ & + & + & + & + & + & + & + & + & + & + & + & + & + & + & - & $b$ \\
\hline \#Eleocharis capillacea Kunth & $\mathrm{EM} / \mathrm{A}$ & - & - & - & - & - & + & - & - & - & + & - & + & - & + & - & c \\
\hline Eleocharis filiculmis Kunth & $\mathrm{EM} / \mathrm{A}$ & - & + & + & + & + & + & - & + & - & + & - & + & + & + & - & $\mathrm{b}$ \\
\hline${ }^{\star}$ Eleocharis geniculata (L.) Roem. \& Schult. & $\mathrm{EM} / \mathrm{A}$ & - & - & - & + & - & + & + & + & - & - & + & - & + & + & - & c \\
\hline Eleocharis interstincta (Vahl) Roem. \& Schult. & EM & + & + & + & + & + & + & + & + & + & + & + & + & + & + & - & $d$ \\
\hline Eleocharis minima Kunth & $\mathrm{EM} / \mathrm{A}$ & - & - & + & + & + & + & + & + & - & - & + & + & + & + & - & $d$ \\
\hline Eleocharis mutata (L.) Roem. \& Schult. & $\mathrm{EM} / \mathrm{A}$ & - & - & - & + & - & + & + & + & - & - & - & + & - & + & - & c \\
\hline "Eleocharis nana Kunth & $\mathrm{EM} / \mathrm{A}$ & - & - & - & - & - & + & - & + & - & - & - & + & - & + & - & c \\
\hline \#Eleocharis retroflexa (Poir.) Urb. & $\mathrm{EM} / \mathrm{A}$ & - & - & + & + & - & + & + & + & - & - & - & - & - & - & - & c \\
\hline Eleocharis sellowiana Kunth & $\mathrm{EM} / \mathrm{A}$ & - & - & - & - & - & - & + & + & - & - & - & - & - & - & - & $d$ \\
\hline${ }^{\#}$ Fimbristylis aestivalis (Retz.) Vahl & $\mathrm{EM} / \mathrm{A}$ & - & - & - & - & + & + & + & + & - & - & + & + & + & - & - & c \\
\hline${ }^{\star}$ Fimbristylis cymosa $\mathrm{R}$. Br. & A & - & - & + & - & - & + & + & + & - & - & - & - & - & - & - & c \\
\hline Fimbristylis dichotoma (L.) Vahl & $\mathrm{EM} / \mathrm{A}$ & + & + & + & + & + & + & + & + & + & + & + & + & + & + & + & c \\
\hline${ }^{\star}$ Fimbristylis miliacea (L.) Vahl & $\mathrm{EM} / \mathrm{A}$ & + & + & + & + & + & + & + & + & + & + & + & + & + & + & - & c \\
\hline Fimbristylis spadicea (L.) Vahl & $\mathrm{EM} / \mathrm{A}$ & - & - & + & + & + & + & + & + & + & - & + & - & + & - & - & c \\
\hline Fuirena umbellata Rottb. & $\mathrm{EM} / \mathrm{A}$ & - & + & + & - & + & + & + & + & - & + & + & + & + & + & - & $b, c$ \\
\hline *Kyllinga odorata Vahl & A & + & - & + & + & + & + & + & + & + & + & + & + & + & + & - & c \\
\hline Lipocarpha humboldtiana Nees & A & + & - & + & - & + & - & + & - & + & - & + & + & + & + & - & c \\
\hline *Lipocarpha salzmanniana Steud. & A & - & - & - & - & + & - & + & - & - & - & + & + & + & - & - & c \\
\hline Oxycaryum cubense (Poepp. \& Kunth) Lye & EM/EP & + & + & + & + & + & + & + & + & + & - & + & + & + & + & - & $d$ \\
\hline *Pycreus polystachyos (Rottb.) P. Beauv. & $\mathrm{EM} / \mathrm{A}$ & - & - & - & - & + & + & + & + & + & - & + & + & + & - & - & $\mathrm{a}, \mathrm{c}$ \\
\hline${ }^{\star}$ Rhynchospora barbata (Vahl) Kunth & EM & - & + & + & + & + & + & + & + & + & + & + & + & + & + & - & $c$ \\
\hline Rhynchospora corymbosa (L.) Britton & EM & - & - & - & - & - & + & - & + & - & + & - & - & - & + & + & $d$ \\
\hline *Rhynchospora emaciata (Nees) Boeckeler & EM & + & - & + & - & + & + & + & + & + & - & + & + & + & + & - & $\mathrm{a}, \mathrm{c}$ \\
\hline
\end{tabular}


Table 2. (Continuation)

\begin{tabular}{|c|c|c|c|c|c|c|c|c|c|c|c|c|c|c|c|c|c|}
\hline \multirow{2}{*}{ Family/ Species } & \multirow{2}{*}{ LF } & \multicolumn{2}{|c|}{$A C$} & \multicolumn{2}{|c|}{ AP } & \multicolumn{2}{|c|}{ AM } & \multicolumn{2}{|c|}{ PA } & \multicolumn{2}{|c|}{ R0 } & \multicolumn{2}{|c|}{$\mathrm{RR}$} & \multicolumn{2}{|c|}{ TO } & \multirow{2}{*}{ END } & \multirow{2}{*}{$\mathrm{HAB}$} \\
\hline & & $\mathrm{F}$ & S & $\mathrm{F}$ & S & $\mathrm{F}$ & S & $\mathrm{F}$ & S & $\mathrm{F}$ & S & $\mathrm{F}$ & S & $\mathrm{F}$ & $S$ & & \\
\hline *Rhynchospora globosa (Kunth) Roem \& Schult. & $\mathrm{EM} / \mathrm{A}$ & + & + & + & + & + & + & + & + & + & + & + & + & + & + & - & $\mathrm{a}, \mathrm{c}$ \\
\hline Rhynchospora holoschoenoides (Rich.) Herter & $\mathrm{EM} / \mathrm{A}$ & + & - & + & + & + & + & + & + & + & + & + & + & + & + & - & $\mathrm{a}, \mathrm{c}$ \\
\hline *Rhynchospora nervosa (Vahl) Boeckeler & EM & + & + & + & + & + & + & + & + & + & + & + & + & + & + & + & c \\
\hline *Rhynchospora schomburgkiana (Boeckeler) T. Koyama & EM & + & - & + & - & + & + & + & - & + & + & + & - & + & - & - & $c$ \\
\hline *Rhynchospora subplumosa C.B. Clarke & EM & + & - & + & - & + & - & + & + & + & - & + & + & + & + & - & $c$ \\
\hline Rhynchospora velutina (Kunth) Boeckeler & EM & + & - & + & + & + & + & + & - & + & - & + & + & + & + & - & $c$ \\
\hline *Scleria microcarpa Nees ex Kunth & A & + & + & + & + & + & + & + & + & + & - & + & + & + & + & - & $c$ \\
\hline *Scleria secans (L.) Urb. & EM & + & - & + & + & + & + & + & + & + & - & + & + & + & + & - & $c$ \\
\hline \multicolumn{18}{|l|}{ Dioscoreaceae } \\
\hline *Dioscorea marginata Griseb. & A & - & - & - & + & + & + & + & + & - & + & - & - & + & + & - & c \\
\hline \multicolumn{18}{|l|}{ Eriocaulaceae } \\
\hline Eriocaulon humboldtii Kunth & $A$ & - & - & + & + & + & - & - & - & - & - & + & + & + & + & - & a \\
\hline \#Paepalanthus bifidus (Schrad.) Kunth & A & - & - & - & - & - & + & + & + & + & - & + & - & - & - & - & $c$ \\
\hline \#Paepalanthus lamarckii Kunth & A & + & + & + & + & - & + & + & + & - & + & - & + & + & + & - & $c$ \\
\hline *Syngonanthus anomalus Ruhland & $\mathrm{EM} / \mathrm{RS}$ & - & - & + & - & + & + & + & + & - & - & + & + & - & - & - & $c$ \\
\hline *Syngonanthus caulescens (Poir.) Ruhland & EM & - & + & - & + & + & + & + & + & - & + & + & + & + & + & - & c \\
\hline "Syngonanthus gracilis (Bong.) Ruhland & EM & - & - & - & - & + & + & + & + & + & + & + & + & - & + & - & c \\
\hline \#Syngonanthus oblongus (Körn.) Ruhland & EM & - & - & - & - & + & + & + & + & - & - & - & - & - & + & - & $c$ \\
\hline Tonina fluviatilis Aubl. & RS & + & + & - & + & - & + & - & + & - & + & - & + & - & + & - & $c$ \\
\hline \multicolumn{18}{|l|}{ Euphorbiaceae } \\
\hline *Acalypha arvensis Poepp. & A & + & + & - & - & - & + & + & + & + & + & - & - & - & + & - & c \\
\hline *Acalypha poiretii Spreng. & $A$ & - & - & - & - & - & + & + & + & - & - & - & - & - & - & - & $\mathrm{a}, \mathrm{c}$ \\
\hline *Astraea lobata (L.) Klotzsch & $A$ & + & - & + & + & + & + & + & + & + & + & + & + & + & + & - & $a, c$ \\
\hline Caperonia castaneifolia (L.) A. St.-Hil. & A & - & - & + & - & + & + & + & + & + & - & + & + & + & + & - & c \\
\hline Croton trinitatis Millsp. & A & + & + & + & + & + & + & + & + & + & + & - & + & + & + & - & c \\
\hline *Dalechampia scandens L. & A & - & + & - & - & + & + & + & + & + & - & - & + & - & - & - & c \\
\hline *Dalechampia tiliifolia Lam. & A & + & + & + & + & - & + & + & + & + & + & + & + & - & + & - & $c$ \\
\hline *Euphorbia hyssopifolia L. & A & + & + & + & + & + & + & + & + & + & + & + & + & + & + & - & c \\
\hline *Euphorbia hirta L. & A & + & + & - & + & + & + & + & + & + & + & + & - & + & + & - & c \\
\hline *Euphorbia thymifolia L. & A & - & - & + & - & + & + & + & + & - & - & - & + & - & - & - & c \\
\hline \multicolumn{18}{|l|}{ Fabaceae } \\
\hline Aeschynomene americana L. & A & + & + & - & - & + & + & + & + & + & + & + & - & + & + & - & $\mathrm{a}, \mathrm{c}$ \\
\hline Aeschynomene fluminensis Vell. & A & + & - & - & + & - & - & + & + & + & - & + & + & + & + & - & $\mathrm{a}, \mathrm{c}$ \\
\hline Aeschynomene paniculata Willd. ex Vogel & A & + & - & + & + & + & + & + & + & + & + & + & + & + & + & - & $\mathrm{a}, \mathrm{c}$ \\
\hline Aeschynomene rudis Benth. & A & - & - & + & + & + & + & + & + & - & - & - & + & - & - & - & $\mathrm{a}, \mathrm{c}$ \\
\hline Aeschynomene sensitiva Sw. & A & - & + & - & + & + & + & + & + & + & + & + & + & - & + & - & c \\
\hline *Calopogonium mucunoides Desv. & A & + & + & + & + & + & + & + & + & + & + & + & + & + & + & - & $c$ \\
\hline *Centrosema brasilianum (L.) Benth. & A & - & - & - & - & + & - & + & - & - & - & + & - & - & - & - & $c$ \\
\hline${ }^{*}$ Chamaecrista calycioides (DC. ex Collad.) Greene & A & - & - & - & - & + & - & + & + & - & - & + & + & - & - & - & c \\
\hline *Chamaecrista diphylla (L.) Greene & A & - & + & + & + & + & + & + & + & + & + & + & + & + & + & - & $c$ \\
\hline *Chamaecrista hispidula (Vahl) H.S.Irwin \& Barneby & A & - & - & - & + & + & + & + & + & - & - & + & + & - & - & - & $c$ \\
\hline Chamaecrista nictitans (L.) Moench & A & + & + & + & + & + & + & + & + & + & + & + & + & + & + & - & c \\
\hline *Clitoria falcata Lam. & $\mathrm{A} / \mathrm{EP}$ & + & + & + & + & + & + & + & + & + & + & + & + & + & + & - & $c$ \\
\hline${ }^{*}$ Clitoria guianensis (Aubl.) Benth. & A/EP & - & - & + & + & + & + & + & + & - & - & + & + & + & + & - & c \\
\hline *Clitoria laurifolia Poir. & $\mathrm{A} / \mathrm{EP}$ & - & + & - & + & + & + & + & + & - & - & + & + & - & - & - & $c$ \\
\hline${ }^{*}$ Crotalaria micans Link & A & + & + & + & + & + & + & + & + & - & + & + & + & - & + & - & $\mathrm{C}$ \\
\hline
\end{tabular}


Table 2. (Continuation)

\begin{tabular}{|c|c|c|c|c|c|c|c|c|c|c|c|c|c|c|c|c|c|}
\hline \multirow{2}{*}{ Family/ Species } & \multirow{2}{*}{ LF } & \multicolumn{2}{|c|}{$A C$} & \multicolumn{2}{|c|}{ AP } & \multicolumn{2}{|c|}{ AM } & \multicolumn{2}{|c|}{ PA } & \multicolumn{2}{|c|}{ RO } & \multicolumn{2}{|c|}{$\mathrm{RR}$} & \multicolumn{2}{|c|}{ TO } & \multirow{2}{*}{ END } & \multirow{2}{*}{$\mathrm{HAB}$} \\
\hline & & $\mathrm{F}$ & $S$ & $\mathrm{~F}$ & $S$ & $\mathrm{~F}$ & $S$ & $\mathrm{~F}$ & $S$ & $\mathrm{~F}$ & $S$ & $\mathrm{~F}$ & $S$ & $\mathrm{~F}$ & $S$ & & \\
\hline${ }^{*}$ Crotalaria nitens Kunth & $A$ & - & - & - & - & + & + & + & + & - & - & - & - & - & + & - & c \\
\hline${ }^{*}$ Crotalaria pallida Aiton & $A$ & + & + & - & + & + & + & + & + & - & + & + & + & - & + & - & c \\
\hline${ }^{*}$ Crotalaria pilosa Mill. & $A$ & - & - & + & + & - & - & + & + & - & - & + & + & + & + & - & c \\
\hline${ }^{\star}$ Crotalaria sagittalis L. & $A$ & - & - & - & - & + & + & - & - & + & - & + & + & - & - & - & c \\
\hline${ }^{*}$ Cymbosema roseum Benth. & $A$ & + & + & + & + & + & + & + & + & - & - & + & + & - & - & - & c \\
\hline *Desmodium barbatum (L.) Benth. & $A$ & + & + & + & + & + & + & + & + & + & + & + & + & + & + & - & c \\
\hline${ }^{*}$ Dioclea guianensis Benth. & $A$ & + & + & + & + & + & + & + & + & - & + & + & + & - & + & - & c \\
\hline *Eriosema simplicifolium (DC.) G. Don & $A$ & - & - & + & + & + & + & + & + & - & - & + & + & + & + & - & c \\
\hline *Indigofera lespedezioides Kunth & A & - & - & - & - & + & + & + & + & + & + & + & + & + & + & - & c \\
\hline *Macrolobium multijugum (DC.) Benth. & $A$ & - & + & + & + & + & + & + & + & - & + & - & + & - & + & - & a \\
\hline *Macroptilium gracile (Poepp. ex Benth.) Urb. & $A$ & - & - & + & - & + & + & + & + & - & - & + & + & - & - & + & c \\
\hline *Mimosa debilis Humb. \& Bonpl. ex Willd. & $A$ & - & - & - & - & + & + & + & + & + & + & + & + & + & + & - & $\mathrm{a}, \mathrm{c}$ \\
\hline `Mimosa dormiens Humb. \& Bonpl. ex Willd. & $A$ & - & - & - & - & + & + & + & + & - & - & - & - & - & - & - & $a, c$ \\
\hline *Mimosa invisa Mart. ex Colla & $A$ & + & - & - & - & + & + & - & - & - & - & + & + & - & - & - & $a, c$ \\
\hline *Mimosa pellita Humb. \& Bonpl. ex Willd. & $A$ & + & - & + & + & + & + & + & + & + & + & + & + & + & + & - & $a, c$ \\
\hline *Mimosa pigra L. & $A$ & + & + & - & + & + & + & - & + & - & + & - & + & - & + & - & $a, c$ \\
\hline *Mimosa pudica L. & $A$ & - & + & - & + & + & + & + & + & + & + & + & + & - & - & - & $a, c$ \\
\hline Mimosa setosa Benth. & $A$ & + & + & - & - & - & - & - & + & - & + & - & + & - & + & - & $a, c$ \\
\hline Neptunia oleracea Lour. & $A$ & - & - & - & - & + & + & + & + & - & - & - & - & - & - & - & $a, c$ \\
\hline *Neptunia plena (L.) Benth. & $A$ & + & - & - & - & + & + & + & + & - & - & - & - & - & - & - & $\mathrm{a}, \mathrm{c}$ \\
\hline *Rhynchosia minima (L.) DC. & $A$ & - & + & - & - & + & + & + & + & - & - & - & + & - & + & - & c \\
\hline Senna obtusifolia (L.) H.S.Irwin \& Barneby & $A$ & + & + & - & - & + & + & + & + & + & + & + & + & + & + & - & c \\
\hline *Senna occidentalis (L.) Link & A & + & + & + & + & + & + & + & + & + & + & + & + & + & - & - & c \\
\hline *Senna reticulata (Willd.) H.S. Irwin \& Barneby & $A$ & + & + & + & + & + & + & + & + & + & + & - & + & - & + & - & c \\
\hline *Sesbania exasperata Kunth & $A$ & + & + & + & + & + & + & + & + & - & + & + & + & - & + & - & c \\
\hline *Stylosanthes guianensis (Aubl.) Sw. & $A$ & - & - & - & + & + & + & + & + & - & + & + & + & + & + & - & c \\
\hline ^Teramnus volubilis Sw. & $A$ & - & - & - & - & + & + & + & + & - & - & - & - & - & - & - & c \\
\hline *Vigna juruana (Harms) Verdc. & $A$ & - & - & - & - & - & + & + & + & - & - & - & + & - & - & - & c \\
\hline Vigna lasiocarpa (Mart.ex Benth.) Verdc. & $A$ & - & - & + & - & + & + & + & + & - & + & + & + & - & - & - & c \\
\hline *Vigna Iongifolia (Benth.) Verdc. & $A$ & - & - & - & - & + & + & + & + & - & + & - & - & - & + & - & c \\
\hline *Zornia guanipensis Pittier & A & - & - & - & + & - & - & + & + & - & - & - & + & - & - & - & c \\
\hline `Zornia latifolia Sm. & A & + & + & + & + & + & + & + & + & + & + & + & + & + & + & - & $a, c$ \\
\hline \multicolumn{18}{|l|}{ Gentianaceae } \\
\hline *Coutoubea ramosa Aubl. & $A$ & + & + & + & + & + & + & + & + & + & + & + & + & + & + & - & $a, c$ \\
\hline *Coutoubea spicata Aubl. & $A$ & + & + & + & + & + & + & + & + & + & + & + & + & + & + & - & c \\
\hline *Schultesia benthamiana Klotzsch & A & - & - & - & - & + & + & + & + & - & - & + & + & - & + & - & c \\
\hline *Schultesia brachyptera Cham. & A & - & - & + & - & + & + & + & + & + & + & + & + & + & + & - & c \\
\hline${ }^{*}$ Schultesia guianensis (Aubl.) Malme & A & + & + & - & - & + & + & + & + & - & - & + & + & - & + & - & c \\
\hline \multicolumn{18}{|l|}{ Gesneriaceae } \\
\hline${ }^{\#}$ Drymonia coccinea (Aubl.) Wiehler & A & + & + & + & + & + & + & + & + & + & + & + & + & - & - & - & c \\
\hline \multicolumn{18}{|l|}{ Haemodoraceae } \\
\hline Schiekia orinocensis (Kunth) Meisn. & A & - & - & - & - & + & + & + & + & + & + & + & + & + & + & - & $\mathrm{a}, \mathrm{c}$ \\
\hline \multicolumn{18}{|l|}{ Heliconiaceae } \\
\hline *Heliconia marginata (Griggs) Pittier & $E M / A$ & + & + & - & + & - & + & - & + & - & - & - & + & - & + & - & $\mathrm{a}, \mathrm{c}$ \\
\hline *Heliconia psittacorum L. f. & $E M / A$ & - & + & + & + & + & + & + & + & + & + & + & + & + & + & - & c \\
\hline Hydrocharitaceae & & & & & & & & & & & & & & & & & \\
\hline
\end{tabular}


Table 2. (Continuation)

\begin{tabular}{|c|c|c|c|c|c|c|c|c|c|c|c|c|c|c|c|c|c|}
\hline \multirow{2}{*}{ Family/ Species } & \multirow{2}{*}{ LF } & \multicolumn{2}{|c|}{$A C$} & \multicolumn{2}{|c|}{ AP } & \multicolumn{2}{|c|}{ AM } & \multicolumn{2}{|c|}{$\mathrm{PA}$} & \multicolumn{2}{|c|}{ RO } & \multicolumn{2}{|c|}{$\mathrm{RR}$} & \multicolumn{2}{|c|}{ TO } & \multirow{2}{*}{ END } & \multirow{2}{*}{$\mathrm{HAB}$} \\
\hline & & $\mathrm{F}$ & $S$ & $\mathrm{~F}$ & $\mathrm{~S}$ & $\mathrm{~F}$ & $S$ & $\mathrm{~F}$ & $S$ & $\mathrm{~F}$ & $S$ & $\mathrm{~F}$ & $S$ & $\mathrm{~F}$ & $S$ & & \\
\hline *Apalanthe granatensis (Bonpl.) Planch. & RS & - & - & - & - & + & - & + & + & - & - & + & + & + & - & - & $b, c$ \\
\hline Najas guadalupensis (Spreng.) Magnus & RS & - & - & + & - & + & - & - & - & - & - & - & - & - & - & - & $a, b$ \\
\hline *Najas microcarpa (A. Braun) Bolle ex Christ & RS & - & - & - & - & + & - & - & - & - & - & - & - & + & + & - & $a, b$ \\
\hline \multicolumn{18}{|l|}{ Hydroleaceae } \\
\hline Hydrolea elatior Schott & A & - & - & - & - & - & - & - & - & - & - & + & + & - & - & - & $c$ \\
\hline Hydrolea spinosa L. & A & + & + & + & + & + & + & + & + & + & + & + & + & + & + & - & $c$ \\
\hline \multicolumn{18}{|l|}{ Hypericaceae } \\
\hline *Vismia cayennensis (Jacq.) Pers. & A & + & + & + & + & + & + & + & + & - & + & + & + & - & - & - & $\mathrm{a}, \mathrm{c}$ \\
\hline \multicolumn{18}{|l|}{ Hypoxidaceae } \\
\hline${ }^{*}$ Curculigo scorzonerifolia (Lam.) Baker & A & - & - & - & + & + & - & + & + & + & + & + & + & - & + & - & $c$ \\
\hline \multicolumn{18}{|l|}{ Iridaceae } \\
\hline${ }^{*}$ Cipura paludosa Aubl. & A & + & + & + & + & + & + & + & + & - & + & + & + & - & + & - & $c$ \\
\hline \multicolumn{18}{|l|}{ Lamiaceae } \\
\hline${ }^{*}$ Hyptis atrorubens Poit. & A & + & + & + & + & + & + & + & + & - & + & + & + & + & + & - & $c$ \\
\hline *Hyptis brevipes Poit. & A & + & + & - & + & + & + & + & + & + & + & - & + & - & + & - & $\mathrm{a}, \mathrm{c}$ \\
\hline${ }^{*}$ Hyptis dilatata Benth. & A & - & - & + & - & + & - & + & - & + & + & + & + & - & + & - & $\mathrm{a}, \mathrm{c}$ \\
\hline *Hyptis lantaniifolia Poit. & A & - & - & - & + & - & + & - & + & - & - & - & + & - & - & - & $\mathrm{a}, \mathrm{c}$ \\
\hline *Hyptis Iorentziana 0. Hoffm. & A & - & + & + & - & + & + & + & + & + & + & - & - & - & - & - & $\mathrm{a}, \mathrm{c}$ \\
\hline *Hyptis mutabilis (Rich.) Briq. & A & + & + & + & + & + & + & + & + & - & + & - & - & + & - & - & $\mathrm{a}, \mathrm{c}$ \\
\hline${ }^{*}$ Hyptis parkeri Benth. & A & - & - & + & - & + & + & + & + & + & + & + & + & - & - & - & c \\
\hline *Hyptis recurvata Poit. & $A$ & + & + & + & + & + & + & + & + & - & - & + & + & - & + & - & $a, c$ \\
\hline *Hyptis spicigera Lam. & $A$ & - & - & - & - & - & + & - & + & - & + & - & + & - & + & - & c \\
\hline${ }^{\star}$ Marsypianthes chamaedrys (Vahl) Kuntze & $A$ & + & + & + & + & + & + & + & + & + & + & + & + & + & + & - & $a, c$ \\
\hline *0cimum campechianum Mill. & A & + & + & - & - & + & + & + & + & - & - & + & + & - & - & - & $c$ \\
\hline *Vitex cymosa Bertero ex Spreng. & A & - & + & - & - & + & + & + & + & + & + & - & - & - & + & - & c \\
\hline Lauraceae & & & & & & & & & & & & & & & & & \\
\hline${ }^{*}$ Cassytha filiformis $\mathrm{L}$. & A & - & - & - & + & + & + & + & + & + & + & + & + & + & + & - & c \\
\hline Lentibulariaceae & & & & & & & & & & & & & & & & & \\
\hline Utricularia breviscapa C. Wright ex Griseb. & FS & + & - & - & + & + & + & + & + & - & - & + & + & + & + & - & $d$ \\
\hline *Utricularia erectiflora A. St.-Hil. \& Girard & A & - & - & - & - & - & - & - & - & - & - & + & + & - & + & - & $\mathrm{a}, \mathrm{c}$ \\
\hline *Utricularia fimbriata Kunth & FS & - & + & - & + & + & + & + & + & - & - & + & + & - & + & - & $a, c$ \\
\hline Utricularia foliosa L. & FS & - & + & + & + & + & + & + & + & - & - & + & + & - & - & - & $d$ \\
\hline Utricularia gibba $\mathrm{L}$. & FS & - & + & - & + & + & + & + & + & - & - & - & + & - & - & - & $d$ \\
\hline *Utricularia guyanensis A. DC. & FS & - & - & - & - & - & - & + & - & - & - & - & + & + & - & - & $\mathrm{a}, \mathrm{c}$ \\
\hline *Utricularia myriocista A. St.-Hil. \& Girard & FS & - & - & + & + & + & + & + & + & - & - & + & + & - & - & - & c \\
\hline *Utricularia pusilla Vahl & A & - & + & - & - & + & + & + & + & - & - & + & + & - & + & - & $c$ \\
\hline *Utricularia simulans Pilg. & $\mathrm{EM} / \mathrm{A}$ & - & - & - & - & + & + & + & + & - & - & + & + & - & + & - & $c$ \\
\hline *Utricularia subulata L. & $\mathrm{EM} / \mathrm{A}$ & - & - & + & + & + & + & + & + & - & + & + & + & + & + & - & $c$ \\
\hline *Utricularia triloba Benj. & $\mathrm{EM} / \mathrm{A}$ & - & - & + & + & + & + & + & + & - & - & + & + & + & - & - & $\mathrm{a}, \mathrm{c}$ \\
\hline Linderniaceae & & & & & & & & & & & & & & & & & \\
\hline Lindernia crustacea (L.) F. Muell. & A & + & + & + & + & + & + & + & + & + & + & + & + & + & + & - & $\mathrm{a}, \mathrm{c}$ \\
\hline Lindernia diffusa (L.) Wettst. & A & + & + & - & - & + & + & + & + & - & + & + & + & - & - & - & $a, c$ \\
\hline \#Torenia thouarsii (Cham. \& Schltdl.) Kuntze & A & + & + & - & - & + & + & + & - & + & + & - & - & - & - & - & c \\
\hline Loganiaceae & & & & & & & & & & & & & & & & & \\
\hline *Spigelia anthelmia L. & A & + & - & - & + & + & + & + & + & + & + & + & + & + & + & - & c \\
\hline Lythraceae & & & & & & & & & & & & & & & & & \\
\hline
\end{tabular}


Table 2. (Continuation)

\begin{tabular}{|c|c|c|c|c|c|c|c|c|c|c|c|c|c|c|c|c|c|}
\hline \multirow{2}{*}{ Family/ Species } & \multirow{2}{*}{ LF } & \multicolumn{2}{|c|}{$\mathrm{AC}$} & \multicolumn{2}{|c|}{ AP } & \multicolumn{2}{|c|}{ AM } & \multicolumn{2}{|c|}{ PA } & \multicolumn{2}{|c|}{ Ro } & \multicolumn{2}{|c|}{$\mathrm{RR}$} & \multicolumn{2}{|c|}{ TO } & \multirow{2}{*}{ END } & \multirow{2}{*}{$\mathrm{HAB}$} \\
\hline & & $\mathrm{F}$ & $\mathrm{S}$ & $\mathrm{F}$ & S & $\mathrm{F}$ & S & $\mathrm{F}$ & $S$ & $\mathrm{~F}$ & $S$ & $\mathrm{~F}$ & S & $\mathrm{F}$ & S & & \\
\hline Cuphea antisyphilitica Kunth & A & - & - & + & + & + & + & + & + & + & + & + & + & + & + & - & $a, c$ \\
\hline Cuphea melvilla Lindl. & A & + & + & + & + & + & + & + & + & + & + & - & + & + & + & - & $a, c$ \\
\hline \multicolumn{18}{|l|}{ Malvaceae } \\
\hline *Byttneria genistella Triana \& Planch. & A & + & + & + & + & + & + & + & + & + & + & - & + & - & + & - & c \\
\hline *Byttneria scabra L. & A & + & - & + & + & - & + & + & + & - & - & - & + & - & - & - & c \\
\hline *Helicteres guazumifolia Kunth & A & - & + & - & - & - & + & - & - & + & + & + & + & - & + & - & $\mathrm{C}$ \\
\hline *Hibiscus bifurcatus Cav. & A & + & + & + & + & - & - & + & + & - & + & - & + & - & - & - & C \\
\hline *Hibiscus dimidiatus Schrank & A & - & - & - & - & - & + & + & + & - & - & - & - & - & - & - & $a, c$ \\
\hline *Hibiscus furcellatus Ders. & A & - & - & + & + & + & + & + & + & + & - & - & + & + & - & - & C \\
\hline *Hibiscus sororius L. & A & + & + & - & - & + & + & - & + & - & - & - & - & - & + & - & c \\
\hline *Melochia arenosa Benth. & A & + & - & + & - & + & + & + & + & - & + & - & + & - & + & - & $a, c$ \\
\hline *Melochia graminifolia A. St.-Hil. & A & + & - & - & - & + & - & + & - & + & + & - & + & + & + & - & $a, c$ \\
\hline Melochia spicata (L.) Fryxell & A & - & - & - & - & - & + & - & - & - & + & - & + & - & + & - & $a, c$ \\
\hline *Pavonia angustifolia Benth. & A & - & - & - & - & - & - & - & - & - & - & + & + & - & + & - & c \\
\hline *Pavonia grandiflora A. St.-Hil. & A & - & - & - & - & - & + & - & - & - & - & + & + & - & + & + & c \\
\hline *Sida acuta Burm. f. & A & - & + & - & - & - & + & + & + & - & + & - & + & + & + & - & $\mathrm{c}$ \\
\hline *Sida rhombifolia L. & A & + & + & + & + & + & + & + & + & + & + & + & + & + & + & - & c \\
\hline${ }^{\star}$ Sida setosa Mart. & A & - & + & - & + & + & + & + & + & - & - & - & + & - & - & - & $\mathrm{c}$ \\
\hline *Urena lobata L. & A & + & + & - & + & + & + & + & + & - & + & - & + & + & + & - & $\mathrm{c}$ \\
\hline \multicolumn{18}{|l|}{ Marantaceae } \\
\hline *Calathea capitata (Ruiz \& Pav.) Lindl. & A & + & + & - & + & + & + & - & + & - & + & - & - & - & + & - & C \\
\hline *Calathea comosa (L. f.) Lindl. & A & + & + & - & - & + & + & - & + & - & - & - & - & - & - & - & $\mathrm{c}$ \\
\hline${ }^{\star}$ Calathea lanata Petersen & A & + & + & - & + & + & + & - & + & - & - & - & - & - & - & - & $\mathrm{C}$ \\
\hline${ }^{\star}$ Calathea loeseneri J.F. Macbr. & A & + & + & - & - & + & + & - & + & - & - & - & - & - & - & - & c \\
\hline${ }^{\star}$ Calathea micans (L. Mathieu) Körn. & A & + & + & + & + & + & + & - & + & + & + & - & + & - & - & - & $\mathrm{C}$ \\
\hline *Ischnosiphon polyphyllus (Poepp. \& Endl.) Körn. & A & - & - & - & - & + & + & + & + & - & - & - & - & - & - & - & $\mathrm{c}$ \\
\hline Thalia geniculata $\mathrm{L}$. & A & + & + & + & + & + & + & + & + & - & + & - & + & - & + & - & $\mathrm{C}$ \\
\hline \multicolumn{18}{|l|}{ Marsileaceae } \\
\hline Marsilea crotophora D.M. Johnson & $\mathrm{RF}$ & - & - & - & - & + & + & - & + & - & - & - & - & - & - & - & $a, c$ \\
\hline Marsilea polycarpa Hook. \& Grev. & $\mathrm{FF}$ & - & + & - & - & - & + & + & + & - & - & + & - & - & - & - & a,c \\
\hline \multicolumn{18}{|l|}{ Mayacaceae } \\
\hline Mayaca fluviatilis Aubl. & $\mathrm{RS}$ & + & + & + & + & + & + & - & + & + & + & - & - & - & - & - & a \\
\hline *Mayaca kunthii Seub. & RS & - & - & - & - & + & - & + & - & - & + & - & - & - & - & - & $a, c$ \\
\hline Mayaca longipes Mart. ex Seub. & $\mathrm{RS}$ & - & - & + & + & + & + & + & + & - & - & - & - & - & - & - & $a, c$ \\
\hline \multicolumn{18}{|l|}{ Melastomataceae } \\
\hline${ }^{*}$ Aciotis acuminifolia (Mart. ex DC.) Triana & A & + & + & + & + & + & + & + & + & + & + & + & + & + & + & - & $\mathrm{C}$ \\
\hline${ }^{\#}$ Aciotis ornata (Miq.) Gleason & A & - & - & + & - & + & + & + & - & - & - & + & - & - & - & - & $\mathrm{C}$ \\
\hline *Acisanthera limnobios (DC.) Triana & A & - & - & - & - & - & - & + & + & - & + & + & + & + & + & - & a,c \\
\hline *Clidemia capitellata (Bonpl.) D. Don & A & + & + & + & + & + & + & + & + & + & + & + & + & + & + & - & C \\
\hline${ }^{\star}$ Comolia villosa (Aubl.) Triana & A & - & - & + & + & + & + & + & + & + & + & + & + & - & - & - & c \\
\hline *Rhynchanthera grandiflora (Aubl.) DC. & A & - & + & + & + & + & + & + & + & + & + & + & + & - & - & - & $a, c$ \\
\hline *Rhynchanthera serrulata (L.C.Rich.) DC. & A & - & + & - & + & + & - & + & + & - & - & + & + & - & + & - & $a, c$ \\
\hline *Tibouchina aspera Aubl. & A & + & + & + & + & + & + & + & + & + & + & - & + & - & - & - & c \\
\hline *Tibouchina gracilis (Bonpl.) Cogn. & A & - & - & - & - & - & - & - & + & - & - & - & + & + & + & - & c \\
\hline Menispermaceae & & & & & & & & & & & & & & & & & \\
\hline *Cissampelos andromorpha DC. & A/EP & + & + & - & - & + & + & + & + & + & + & + & + & + & + & - & a \\
\hline
\end{tabular}


Table 2. (Continuation)

\begin{tabular}{|c|c|c|c|c|c|c|c|c|c|c|c|c|c|c|c|c|c|}
\hline \multirow{2}{*}{ Family/ Species } & \multirow{2}{*}{ LF } & \multicolumn{2}{|c|}{$\mathrm{AC}$} & \multicolumn{2}{|c|}{$\mathrm{AP}$} & \multicolumn{2}{|c|}{ AM } & \multicolumn{2}{|c|}{ PA } & \multicolumn{2}{|c|}{ RO } & \multicolumn{2}{|c|}{$\mathrm{RR}$} & \multicolumn{2}{|c|}{ TO } & \multirow{2}{*}{ END } & \multirow{2}{*}{$\mathrm{HAB}$} \\
\hline & & $\mathrm{F}$ & S & $\mathrm{F}$ & S & $\mathrm{F}$ & S & $\mathrm{F}$ & S & $\mathrm{F}$ & S & $\mathrm{F}$ & $S$ & $\mathrm{~F}$ & S & & \\
\hline *Cissampelos glaberrima A. St.-Hil. & A/EP & + & + & - & - & - & + & - & - & - & - & - & + & - & + & - & $\mathrm{a}, \mathrm{c}$ \\
\hline *Odontocarya arifolia Barneby & A & - & - & - & - & + & + & - & - & - & + & - & - & - & - & - & $a, c$ \\
\hline *Odontocarya tamoides (DC.) Miers & A & - & + & - & - & - & + & - & + & - & - & - & + & + & + & - & $\mathrm{a}, \mathrm{c}$ \\
\hline \multicolumn{18}{|l|}{ Menyanthaceae } \\
\hline Nymphoides indica (L.) Kuntze & RF & - & - & + & + & + & + & + & + & + & - & + & + & + & + & - & d \\
\hline \multicolumn{18}{|l|}{ Molluginaceae } \\
\hline *Mollugo verticillata $\mathrm{L}$. & EM & - & - & - & + & + & + & + & + & - & + & - & + & - & + & - & c \\
\hline \multicolumn{18}{|l|}{ Myrtaceae } \\
\hline *Psidium acutangulum DC. & A & + & + & - & + & + & + & + & + & + & + & + & - & + & + & - & $c$ \\
\hline \multicolumn{18}{|l|}{ Nymphaeaceae } \\
\hline Nymphaea gardneriana Planch. & RF & + & + & - & - & - & - & + & + & - & - & - & + & - & + & - & $\mathrm{a}, \mathrm{c}$ \\
\hline Nymphaea rudgeana G. Mey. & RF & - & - & + & + & + & + & + & + & - & - & - & + & - & - & - & $\mathrm{a}, \mathrm{c}$ \\
\hline Victoria amazonica (Poepp.) J.E. Sowerby & RF & + & + & - & - & + & + & + & + & - & - & - & - & - & - & - & $\mathrm{a}, \mathrm{c}$ \\
\hline \multicolumn{18}{|l|}{ Ochnaceae } \\
\hline *Sauvagesia erecta L. & A & + & + & + & + & + & + & + & + & + & + & + & + & + & + & - & $c$ \\
\hline *Sauvagesia rubiginosa A.St.-Hil. & A & - & + & + & - & + & + & + & + & - & - & + & + & - & - & - & $c$ \\
\hline *Sauvagesia sprengelii A. St.-Hil. & A & + & + & + & + & + & + & + & + & + & + & + & + & - & + & - & $c$ \\
\hline \multicolumn{18}{|l|}{ Onagraceae } \\
\hline *Ludwigia affinis (DC.) H. Hara & EM & + & + & + & + & + & + & + & + & + & + & + & + & - & + & - & $\mathrm{a}, \mathrm{c}$ \\
\hline *Ludwigia decurrens Walter & $\mathrm{EM} / \mathrm{A}$ & + & + & + & + & + & + & + & + & + & + & - & - & - & + & - & $a, c$ \\
\hline *Ludwigia densiflora (Micheli) H. Hara & $\mathrm{EM} / \mathrm{A}$ & + & + & - & - & + & + & + & + & + & + & - & + & - & - & - & $\mathrm{a}, \mathrm{c}$ \\
\hline Ludwigia helminthorrhiza (Mart.) H. Hara & $\mathrm{EM} / \mathrm{FF}$ & - & + & - & - & - & + & + & + & - & + & - & - & - & - & - & $d$ \\
\hline *Ludwigia hyssopifolia (G. Don) Exell & $\mathrm{EM} / \mathrm{A}$ & + & + & - & + & + & + & + & + & + & + & + & + & - & + & - & $\mathrm{a}, \mathrm{c}$ \\
\hline *Ludwigia leptocarpa (Nutt.) H. Hara & EM & + & + & - & + & + & + & + & + & - & + & - & + & - & + & - & $\mathrm{a}, \mathrm{c}$ \\
\hline *Ludwigia nervosa (Poir.) H. Hara & EM/A & - & - & + & + & + & + & + & + & + & + & + & + & + & + & - & $\mathrm{a}, \mathrm{c}$ \\
\hline Ludwigia octovalvis (Jacq.) P.H. Raven & $\mathrm{EM} / \mathrm{A}$ & + & + & - & + & + & + & + & + & + & + & - & + & + & + & - & $a, c$ \\
\hline *Ludwigia rigida (Miq.) Sandwith & $\mathrm{EM} / \mathrm{A}$ & - & - & + & + & - & - & + & + & + & - & + & + & - & - & - & $a, c$ \\
\hline Ludwigia sedoides (Humb. \& Bonpl.) H. Hara & FF & + & + & + & - & + & + & + & + & + & + & + & + & - & + & - & $d$ \\
\hline *Ludwigia tomentosa (Cambess.) H. Hara & $\mathrm{EM} / \mathrm{A}$ & - & - & - & - & - & - & - & + & - & - & - & - & + & + & - & $\mathrm{a}, \mathrm{c}$ \\
\hline *Ludwigia torulosa (Arn.) H. Hara & $\mathrm{EM} / \mathrm{A}$ & - & - & + & + & - & - & + & + & + & + & - & - & - & - & - & $\mathrm{a}, \mathrm{c}$ \\
\hline
\end{tabular}
Orchidaceae

\begin{tabular}{|c|c|c|c|c|c|c|c|c|c|c|c|c|c|c|c|c|c|}
\hline${ }^{\star}$ Eulophia alta (L.) Fawc. \& Rendle & A & - & + & + & - & + & + & + & + & + & - & - & - & + & + & - & C \\
\hline \multicolumn{18}{|l|}{ Orobanchaceae } \\
\hline *Buchnera palustris (Aubl.) Spreng. & A & + & + & + & + & + & + & + & + & + & + & + & + & + & + & - & $\mathrm{c}$ \\
\hline${ }^{\star}$ Buchnera rosea Kunth & A & + & - & - & - & + & + & + & + & + & + & + & + & + & + & - & $a, c$ \\
\hline Melasma melampyroides (Rich.) Pennell & A & - & - & - & - & + & + & - & - & - & - & - & - & - & - & - & $c$ \\
\hline \multicolumn{18}{|l|}{ Oxalidaceae } \\
\hline *Oxalis barrelieri L. & A & + & + & - & - & + & + & + & + & - & + & + & + & - & - & - & $\mathrm{a}, \mathrm{c}$ \\
\hline \multicolumn{18}{|l|}{ Passifloraceae } \\
\hline *Passiflora foetida L. & EP & + & + & + & + & + & + & + & + & + & + & + & + & + & + & - & $a, c$ \\
\hline *Passiflora vespertilio L. & A & + & + & + & + & + & + & + & + & + & + & - & + & - & - & - & $\mathrm{a}, \mathrm{c}$ \\
\hline *Piriqueta cistoides (L.) Griseb. & A & + & + & + & + & + & + & + & + & + & + & + & + & + & + & - & $\mathrm{c}$ \\
\hline *Piriqueta duarteana (A. St.-Hil., A. Juss. \& Cambess.) Urb. & A & - & - & - & - & - & - & + & + & - & - & - & - & + & + & + & $\mathrm{c}$ \\
\hline *Turnera scabra Millsp. & A & + & + & + & - & + & + & + & - & - & - & + & + & - & - & - & $\mathrm{C}$ \\
\hline \multicolumn{18}{|l|}{ Pedaliaceae } \\
\hline "Sesamum indicum L. & A & - & + & - & + & - & + & - & + & - & - & - & - & - & - & - & $\mathrm{c}$ \\
\hline
\end{tabular}


Table 2. (Continuation)

\begin{tabular}{|c|c|c|c|c|c|c|c|c|c|c|c|c|c|c|c|c|c|}
\hline \multirow{2}{*}{ Family/ Species } & \multirow{2}{*}{ LF } & \multicolumn{2}{|c|}{$\mathrm{AC}$} & \multicolumn{2}{|c|}{ AP } & \multicolumn{2}{|c|}{ AM } & \multicolumn{2}{|c|}{ PA } & \multicolumn{2}{|c|}{ RO } & \multicolumn{2}{|c|}{$\mathrm{RR}$} & \multicolumn{2}{|c|}{ TO } & \multirow{2}{*}{ END } & \multirow{2}{*}{$\mathrm{HAB}$} \\
\hline & & $\mathrm{F}$ & S & $\mathrm{F}$ & $\mathrm{S}$ & $\mathrm{F}$ & S & $\mathrm{F}$ & $S$ & $\mathrm{~F}$ & $S$ & $\mathrm{~F}$ & S & $\mathrm{F}$ & $S$ & & \\
\hline \multicolumn{18}{|l|}{ Phyllanthaceae } \\
\hline Phyllanthus fluitans Benth. ex Müll. Arg. & $\mathrm{FF}$ & - & - & + & + & + & + & + & + & - & - & - & - & - & - & - & $\mathrm{a}, \mathrm{c}$ \\
\hline *Phyllanthus niruri L. & EM & + & + & + & + & + & + & + & + & + & + & + & + & + & + & - & $\mathrm{a}, \mathrm{c}$ \\
\hline *Phyllanthus stipulatus (Raf.) G.L.Webster & $\mathrm{FF}$ & + & + & - & - & + & + & + & + & + & + & + & + & - & - & - & $\mathrm{a}, \mathrm{c}$ \\
\hline \multicolumn{18}{|l|}{ Plantaginaceae } \\
\hline *Bacopa aquatica Aubl. & RS & - & - & + & - & + & + & + & + & - & - & - & - & - & - & - & $\mathrm{a}, \mathrm{c}$ \\
\hline Bacopa arenaria Loefgr. \& Edwall & EM & - & - & - & - & - & + & + & + & - & - & - & - & - & - & + & b \\
\hline \#Bacopa egensis (Poepp.) Pennell & EM & - & + & - & - & + & + & + & + & - & - & - & - & - & - & - & c \\
\hline Bacopa reflexa (Benth.) Edwall & EM & + & - & + & - & + & - & + & + & - & - & + & + & - & + & - & $a, c$ \\
\hline *Bacopa serpyllifolia (Benth.) Pennell & RS & + & - & - & - & - & + & - & - & - & - & + & + & - & - & - & $a, c$ \\
\hline *Conobea aquatica Aubl. & EM/A & - & - & + & + & - & - & + & + & - & - & + & + & - & - & - & $a, c$ \\
\hline *Mecardonia procumbens (Mill.) Small & A & + & + & - & + & + & + & + & - & - & - & - & - & - & - & - & a \\
\hline *Scoparia dulcis L. & A & + & + & + & + & + & + & + & + & + & + & + & + & + & + & - & $a, c$ \\
\hline \multicolumn{18}{|l|}{ Poaceae } \\
\hline *Andropogon bicornis L. & $\mathrm{EM} / \mathrm{A}$ & + & + & + & + & + & + & + & + & + & + & + & + & + & + & - & $a, c$ \\
\hline *Andropogon selloanus (Hack.) Hack. & $\mathrm{EM} / \mathrm{A}$ & - & - & - & - & + & + & + & + & - & - & + & + & + & + & - & $\mathrm{a}, \mathrm{c}$ \\
\hline *Andropogon virgatus Desv. & $\mathrm{EM} / \mathrm{A}$ & - & - & + & + & + & + & + & + & - & - & - & + & + & + & - & $\mathrm{a}, \mathrm{c}$ \\
\hline *Axonopus leptostachyus (Flüggé) Hitchc. & A & + & - & + & + & + & + & + & + & - & - & + & + & - & - & - & $\mathrm{a}, \mathrm{c}$ \\
\hline${ }^{\star}$ Axonopus purpusii (Mez) Chase & A & + & - & + & + & - & - & + & + & - & + & + & + & - & + & - & $a, c$ \\
\hline${ }^{\star}$ Cenchrus purpureus (Schumach.) Morrone & $\mathrm{EM} / \mathrm{A}$ & - & - & + & - & + & + & - & - & - & - & - & + & - & - & - & c \\
\hline *Coix lacryma-jobi L. & A & + & + & - & - & - & + & - & + & - & + & - & - & - & - & - & $a, c$ \\
\hline *Cynodon dactylon (L.) Pers. & A & - & - & - & - & + & + & + & + & + & - & - & - & - & - & - & $a, c$ \\
\hline *Digitaria ciliaris (Retz.) Koeler & A & - & - & - & - & + & + & + & + & - & - & - & - & - & - & - & $a, c$ \\
\hline *Digitaria horizontalis Willd. & A & + & + & + & + & + & + & + & + & - & - & - & - & + & + & - & a,c \\
\hline *Digitaria violascens Link & A & - & - & + & - & + & - & + & + & - & - & + & - & - & - & - & $\mathrm{a}, \mathrm{c}$ \\
\hline *Dinebra scabra (Nees) P.M. Peterson \& N. Snow & $\mathrm{EM} / \mathrm{A}$ & - & - & - & - & + & - & + & - & + & - & - & - & - & - & - & a,c \\
\hline Echinochloa colona (L.) Link & EM & - & - & + & - & + & + & + & + & + & + & - & + & - & + & - & a,c \\
\hline Echinochloa crusgalli (L.) P. Beauv. & EM & - & - & - & - & + & + & + & - & - & - & + & - & + & - & - & a,c \\
\hline Echinochloa crus-pavonis (Kunth) Schult. & EM & + & - & - & - & + & - & + & + & - & - & - & + & - & + & - & c \\
\hline Echinochloa polystachya (Kunth) Hitchc. & $\mathrm{EM} / \mathrm{RF}$ & - & + & - & - & + & + & + & + & - & - & - & - & - & - & - & $\mathrm{a}, \mathrm{c}$ \\
\hline *Eleusine indica (L.) Gaertn. & A & + & + & + & + & + & + & + & + & + & + & + & + & + & + & - & c \\
\hline *Eragrostis acutiflora (Kunth) Nees & A & - & - & + & + & + & - & + & + & + & - & - & - & - & - & - & $a, c$ \\
\hline${ }^{\star}$ Eragrostis ciliaris (L.) R. Br. & A & + & - & + & + & + & + & + & + & + & - & + & + & - & - & - & $a, c$ \\
\hline *Eragrostis japonica (Thunb.) Trin. & A & - & - & + & - & + & - & + & + & + & - & + & + & - & - & - & a,c \\
\hline *Eragrostis hypnoides (Lam.) Britton, Sterns \& Poggenb. & A & + & + & + & + & + & + & + & + & + & + & + & + & - & + & + & c \\
\hline *Eragrostis pilosa (L.) P. Beauv. & A & - & - & + & + & - & + & + & + & - & - & + & - & - & - & - & a,c \\
\hline *Eragrostis tenella (L.) P. Beauv. ex Roem. \& Schult. & A & - & - & + & - & + & + & + & - & - & + & + & - & - & - & - & a,c \\
\hline *Eriochloa distachya Kunth & A & - & - & - & - & - & - & + & - & - & + & - & - & - & + & - & c \\
\hline *Eriochrysis cayennensis P. Beauv. & $A$ & - & - & + & + & - & + & + & + & - & - & + & + & - & - & - & c \\
\hline Gynerium sagittatum (Aubl.) P. Beauv. & $A$ & - & - & - & - & + & + & - & + & - & + & - & + & - & - & - & $\mathrm{a}, \mathrm{c}$ \\
\hline *Homolepis aturensis (Kunth) Chase & A & + & + & + & + & + & + & + & + & + & + & + & + & - & - & + & $a, c$ \\
\hline Hymenachne amplexicaulis (Rudge) Nees & $\mathrm{EM} / \mathrm{A}$ & - & - & - & + & + & + & + & + & - & + & - & + & - & + & - & $\mathrm{a}, \mathrm{c}$ \\
\hline Hymenachne donacifolia (Raddi) Chase & $\mathrm{EM} / \mathrm{A}$ & + & + & - & - & - & + & - & + & - & + & - & - & - & - & - & $a, c$ \\
\hline */sachne polygonoides (Lam.) Döll & $\mathrm{EM} / \mathrm{A}$ & + & + & + & - & + & + & + & + & + & - & - & + & + & + & + & c \\
\hline *Ischaemum guianense Kunth ex Hack. & $\mathrm{EM} / \mathrm{A}$ & - & - & + & + & - & - & - & + & + & - & - & - & - & - & - & a,c \\
\hline *Lasiacis procerrima (Hack.) Hitchc. & $\mathrm{EM} / \mathrm{A}$ & - & - & - & - & + & + & - & - & - & - & + & + & - & - & - & a,c \\
\hline
\end{tabular}


Table 2. (Continuation)

\begin{tabular}{|c|c|c|c|c|c|c|c|c|c|c|c|c|c|c|c|c|c|}
\hline \multirow{2}{*}{ Family/ Species } & \multirow{2}{*}{ LF } & \multicolumn{2}{|c|}{$A C$} & \multicolumn{2}{|c|}{ AP } & \multicolumn{2}{|c|}{ AM } & \multicolumn{2}{|c|}{ PA } & \multicolumn{2}{|c|}{ RO } & \multicolumn{2}{|c|}{$\mathrm{RR}$} & \multicolumn{2}{|c|}{ TO } & \multirow{2}{*}{ END } & \multirow{2}{*}{ HAB } \\
\hline & & $\mathrm{F}$ & S & $\mathrm{F}$ & $S$ & $\mathrm{~F}$ & $S$ & $\mathrm{~F}$ & $S$ & $\mathrm{~F}$ & $S$ & $\mathrm{~F}$ & S & $\mathrm{F}$ & $S$ & & \\
\hline Leersia hexandra Sw. & $E M / A$ & - & - & + & + & + & + & + & + & - & - & - & - & - & - & - & $\mathrm{a}$ \\
\hline *Leptochloa virgata (L.) P. Beauv. & EM/A & - & - & + & - & + & + & + & + & - & - & + & - & + & + & - & $a, c$ \\
\hline Luziola spruceana Benth. ex Döll & EM/A & + & - & + & - & + & + & + & + & - & - & - & - & - & - & - & $\mathrm{a}, \mathrm{c}$ \\
\hline Luziola subintegra Swallen & EM/A & - & - & + & - & - & - & + & + & - & - & + & + & - & + & - & $a, c$ \\
\hline *Megathyrsus maximus (Jacq.) BK Simon \& SWL Jacobs & EM & - & - & - & - & + & + & - & + & - & - & - & - & - & - & - & $\mathrm{a}, \mathrm{c}$ \\
\hline *Mesosetum Ioliiforme (Hochst. ex Steud.) Chase & A & + & + & + & + & + & + & + & + & + & - & + & + & + & + & - & $\mathrm{a}, \mathrm{c}$ \\
\hline *Olyra ecaudata Döll & A & + & + & - & + & + & - & + & + & - & + & - & + & - & - & - & $\mathrm{a}, \mathrm{c}$ \\
\hline *Olyra longifolia Kunth & A & - & + & + & + & + & + & + & + & - & - & + & + & - & - & - & $\mathrm{a}, \mathrm{c}$ \\
\hline Oryza grandiglumis (Döll) Prod. & A & + & + & - & - & + & + & + & + & - & - & - & - & - & + & - & $\mathrm{a}, \mathrm{c}$ \\
\hline Oryza latifolia Desv. & A & - & - & + & + & + & + & + & + & - & - & - & - & - & + & - & $a, c$ \\
\hline Oryza sativa L. & A & + & - & + & - & + & - & + & + & + & - & + & - & + & + & - & $\mathrm{a}, \mathrm{c}$ \\
\hline *Otachyrium succisum (Swallen) Send. \& Soderstr. & A & - & + & - & - & - & - & + & + & - & - & - & + & - & - & - & $\mathrm{a}, \mathrm{c}$ \\
\hline *Otachyrium versicolor (Döll) Henrard & A & + & + & - & - & + & + & + & + & - & - & - & + & - & - & - & a \\
\hline *Panicum aquaticum Poir. & EM & + & - & - & - & + & - & + & + & - & - & - & - & - & - & - & $\mathrm{a}, \mathrm{c}$ \\
\hline *Panicum dichotomiflorum Michx. & EM & - & - & + & - & + & + & + & + & - & - & + & - & + & + & - & $\mathrm{a}, \mathrm{c}$ \\
\hline Panicum elephantipes Nees ex Trin. & EM & - & - & + & + & + & + & + & + & + & - & + & + & - & - & - & $\mathrm{a}, \mathrm{c}$ \\
\hline *Panicum grande Hitchc. \& Chase & EM & - & - & - & - & - & - & + & + & - & - & - & - & - & - & - & $\mathrm{a}, \mathrm{c}$ \\
\hline Panicum repens $\mathrm{L}$. & EM & - & - & - & - & + & - & + & - & - & - & - & - & - & - & - & $\mathrm{a}, \mathrm{c}$ \\
\hline Paratheria prostrata Griseb. & A & - & - & - & - & + & - & + & + & - & - & - & - & - & - & - & $c$ \\
\hline *Pariana campestris Aubl. & A & - & - & + & + & - & + & + & + & - & + & - & + & - & + & - & $a, c$ \\
\hline *Paspalum boscianum Flüggé & EM & + & + & + & - & + & + & + & + & + & + & + & + & + & - & - & $\mathrm{a}, \mathrm{c}$ \\
\hline${ }^{\star}$ Paspalum conjugatum P.J. Bergius & EM & + & + & + & + & + & + & + & + & + & + & + & + & + & + & - & $\mathrm{a}, \mathrm{c}$ \\
\hline *Paspalum conspersum Schrad. & EM & - & - & - & - & - & - & + & + & - & + & - & - & - & + & - & $\mathrm{a}, \mathrm{c}$ \\
\hline *Paspalum coryphaeum Trin. & EM & - & - & - & + & - & + & + & - & - & - & + & + & + & - & - & $\mathrm{a}, \mathrm{c}$ \\
\hline *Paspalum densum Poir. & EM & - & - & - & - & - & - & + & + & - & - & + & + & + & + & - & $\mathrm{a}, \mathrm{c}$ \\
\hline *Paspalum denticulatum Trin. & EM & - & - & - & - & - & - & + & - & - & - & - & - & - & - & - & a \\
\hline Paspalum fasciculatum Willd. ex Flüggé & EM & - & + & + & - & + & + & + & + & - & + & + & - & - & - & - & $\mathrm{a}, \mathrm{c}$ \\
\hline *Paspalum ligulare Nees & EM & - & - & - & - & - & - & + & - & - & - & - & - & - & - & - & $\mathrm{a}, \mathrm{c}$ \\
\hline *Paspalum melanospermum Desv. ex Poir. & EM & + & + & + & + & + & + & + & + & + & + & + & + & + & + & - & $\mathrm{a}, \mathrm{c}$ \\
\hline *Paspalum orbiculatum Poir. & EM & - & - & + & + & + & + & + & + & - & + & + & - & - & - & - & $\mathrm{a}, \mathrm{c}$ \\
\hline *Paspalum plicatulum Michx. & EM & - & - & - & - & - & + & + & + & - & - & + & + & + & + & - & $\mathrm{a}, \mathrm{c}$ \\
\hline *Paspalum pulchellum Kunth & EM & - & - & + & + & + & - & + & + & - & - & + & + & - & + & - & $\mathrm{a}, \mathrm{c}$ \\
\hline Paspalum repens P.J. Bergius & $\mathrm{EM} / \mathrm{FF}$ & + & + & + & - & + & + & + & + & + & + & + & + & + & + & - & $a, c$ \\
\hline *Paspalum trichophyllum Henrard & EM & - & - & - & - & - & - & + & - & - & - & - & - & - & - & - & $a, c$ \\
\hline Paspalum vaginatum Sw. & EM & - & - & - & - & - & + & + & + & - & - & - & - & - & - & - & $a, c$ \\
\hline Paspalum virgatum $\mathrm{L}$. & EM & + & + & + & + & + & + & + & + & + & + & + & + & + & + & - & $a, c$ \\
\hline *Paspalum wrightii Hitchc. \& Chase & EM & - & - & - & - & - & - & + & - & + & - & - & - & + & - & - & $\mathrm{a}, \mathrm{c}$ \\
\hline *Raddiella esenbeckii (Steud.) Calderón \& Soderstr. & A & - & - & - & - & + & + & + & + & - & + & + & + & - & + & - & $\mathrm{a}, \mathrm{c}$ \\
\hline *Reimarochloa aberrans (Döll) Chase & A & - & - & - & - & - & - & + & + & - & - & - & - & - & - & - & $\mathrm{a}, \mathrm{c}$ \\
\hline *Reimarochloa acuta (Flüggé) Hitchc. & A & - & - & - & + & + & + & + & + & - & - & - & - & + & - & - & $\mathrm{a}, \mathrm{c}$ \\
\hline *Reimarochloa brasiliensis (Spreng.) Hitchc. & A & - & - & - & - & + & + & + & - & - & - & - & - & + & - & - & $\mathrm{a}, \mathrm{c}$ \\
\hline *Rugoloa hylaeica (Mez) Zuloaga & EM & - & - & - & - & - & - & - & - & - & - & - & - & + & - & - & $\mathrm{a}, \mathrm{c}$ \\
\hline${ }^{\star}$ Rugoloa pilosa (Sw). Zuloaga & EM & + & - & + & - & + & - & + & - & + & - & + & - & + & - & - & $\mathrm{a}, \mathrm{c}$ \\
\hline *Rugoloa polygonata (Schrad.) Zuloaga & EM & + & - & + & - & - & - & + & - & - & - & - & - & - & - & + & $\mathrm{a}, \mathrm{c}$ \\
\hline *Sacciolepis myuros (Lam.) Chase & A & - & - & - & + & + & + & - & + & - & + & - & + & - & + & - & $\mathrm{a}, \mathrm{c}$ \\
\hline${ }^{\star}$ Sacciolepis striata (L.) Nash & A & - & - & + & + & - & - & - & - & - & - & - & - & - & - & - & $a, c$ \\
\hline
\end{tabular}


Table 2. (Continuation)

\begin{tabular}{|c|c|c|c|c|c|c|c|c|c|c|c|c|c|c|c|c|c|}
\hline \multirow{2}{*}{ Family/ Species } & \multirow{2}{*}{ LF } & \multicolumn{2}{|c|}{ AC } & \multicolumn{2}{|c|}{ AP } & \multicolumn{2}{|c|}{ AM } & \multicolumn{2}{|c|}{ PA } & \multicolumn{2}{|c|}{ RO } & \multicolumn{2}{|c|}{$\mathrm{RR}$} & \multicolumn{2}{|c|}{ TO } & \multirow{2}{*}{ END } & \multirow{2}{*}{ HAB } \\
\hline & & $\mathrm{F}$ & S & $\mathrm{F}$ & $S$ & $\mathrm{~F}$ & S & $\mathrm{F}$ & S & $\mathrm{F}$ & S & $\mathrm{F}$ & S & $\mathrm{F}$ & S & & \\
\hline *Setaria parviflora (Poir.) Kerguélen & EM & + & + & + & + & + & + & + & + & - & + & + & + & + & + & - & $a, c$ \\
\hline *Sorghastrum setosum (Griseb.) Hitchc. & EM & - & - & - & - & + & + & + & + & - & - & + & + & - & - & - & $a, c$ \\
\hline *Sorghum bicolor subsp. arundinaceum (Desv.) de Wet \& J.R.Harlan & EM & + & + & - & - & - & - & - & + & - & - & - & - & - & - & - & $a, c$ \\
\hline *Sporobolus virginicus (L.) Kunth & EM & - & - & - & + & - & - & + & + & - & - & - & - & - & - & - & $a, c$ \\
\hline *Steinchisma laxum (Sw.) Zuloaga & EM & + & + & + & + & + & + & + & + & - & - & - & - & + & + & - & c \\
\hline *Stephostachys mertensii (Roth) Zuloaga \& Morrone & EM & + & - & - & - & + & - & + & - & - & - & + & - & - & - & - & a \\
\hline *Trichanthecium cyanescens (Nees ex Trin.) Zuloaga \& Morrone & EM & - & - & + & - & + & + & + & - & + & + & + & + & + & - & - & $a, c$ \\
\hline Trichanthecium micranthum (Kunth) Zuloaga \& Morrone & EM & + & - & - & - & + & - & + & - & + & - & + & - & - & - & - & $\mathrm{a}, \mathrm{c}$ \\
\hline *Trichanthecium nervosum (Lam.) Zuloaga \& Morrone & EM & - & - & + & + & + & + & + & - & + & + & + & - & - & - & - & $\mathrm{a}, \mathrm{c}$ \\
\hline *Trichanthecium parvifolium (Lam.) Zuloaga \& Morrone & EM & + & + & + & - & + & + & + & - & + & - & + & + & + & - & - & $\mathrm{a}, \mathrm{c}$ \\
\hline *Trichanthecium pyrularium (Hitchc. \& Chase) Zuloaga \& Morrone & EM & - & - & + & - & + & - & + & + & + & - & - & - & - & - & - & $\mathrm{a}, \mathrm{c}$ \\
\hline \multicolumn{18}{|l|}{ Podostemaceae } \\
\hline${ }^{\#}$ Apinagia aripecurensis P. Royen & $\mathrm{EM} / \mathrm{RS}$ & - & - & - & - & - & - & + & - & - & - & - & - & - & - & + & $a, c$ \\
\hline \#Apinagia batrachifolia (Mildbr.) P. Royen var. batrachifolia & $\mathrm{EM} / \mathrm{RS}$ & - & - & + & + & + & + & - & - & - & - & - & - & - & - & + & $a, c$ \\
\hline $\begin{array}{l}\text { \#Apinagia corymbosa (Engl.) P. Royen var. capillarifolia (Engl.) P. } \\
\text { Royen }\end{array}$ & $\mathrm{EM} / \mathrm{RS}$ & - & - & - & - & - & - & - & - & - & - & + & - & - & + & + & $\mathrm{a}, \mathrm{c}$ \\
\hline \#Apinagia corymbosa (Tul.) Engler var. corymbosa & $\mathrm{EM} / \mathrm{RS}$ & - & - & - & - & - & - & + & - & - & - & - & - & + & + & + & $a, c$ \\
\hline \#Apinagia exlilis (Tul.) P. Royen & $\mathrm{EM} / \mathrm{RS}$ & - & - & - & - & + & - & + & - & - & - & - & - & - & - & - & $a, c$ \\
\hline \#Apinagia fimbrifolia P. Royen & $\mathrm{EM} / \mathrm{RS}$ & - & - & - & - & + & + & - & - & + & - & - & - & + & + & - & $\mathrm{a}, \mathrm{c}$ \\
\hline${ }^{\#}$ Apinagia guyanensis (Pulle) P. Roeyn & $\mathrm{EM} / \mathrm{RS}$ & - & - & + & + & + & + & + & + & - & - & - & - & - & - & - & $a, c$ \\
\hline \#Apinagia kockii (Engler) P. Royen & $\mathrm{EM} / \mathrm{RS}$ & - & - & - & - & - & - & - & - & - & - & + & - & - & - & - & $\mathrm{a}, \mathrm{c}$ \\
\hline \#Apinagia longifolia (Tul.) P. Royen & EM/RS & - & - & - & + & - & - & + & - & - & - & - & + & - & - & - & $\mathrm{a}, \mathrm{c}$ \\
\hline \#Apinagia minor P. Royen & $\mathrm{EM} / \mathrm{RS}$ & - & - & - & - & - & - & + & - & - & - & - & - & - & - & - & $a, c$ \\
\hline "Apinagia platystigma P. Royen & $\mathrm{EM} / \mathrm{RS}$ & - & - & + & - & - & + & - & - & - & - & - & - & - & - & - & $\mathrm{a}, \mathrm{c}$ \\
\hline \#Apinagia rangiferina P. Royen & $\mathrm{EM} / \mathrm{RS}$ & - & - & - & - & + & + & - & - & - & - & - & - & - & - & - & $a, c$ \\
\hline "Apinagia richardiana (Tul.) P. Royen & $\mathrm{EM} / \mathrm{RS}$ & - & - & + & + & + & + & + & + & - & - & + & + & - & + & - & $a, c$ \\
\hline${ }^{\#}$ Apinagia secundiflora (Tul.) Pulle & $\mathrm{EM} / \mathrm{RS}$ & - & - & - & + & - & - & - & - & - & - & - & - & - & - & - & $\mathrm{a}, \mathrm{c}$ \\
\hline${ }^{\#}$ Apinagia spruceana (Wedd.) Engler & $\mathrm{EM} / \mathrm{RS}$ & - & - & - & - & + & - & - & - & - & - & - & - & - & - & - & $a, c$ \\
\hline \#Apinagia surumuensis (Engler) P. Royen & $\mathrm{EM} / \mathrm{RS}$ & - & - & - & - & - & + & - & - & - & - & + & - & - & - & - & $\mathrm{a}, \mathrm{c}$ \\
\hline \#Apinagia tenuifolia P. Royen & $\mathrm{EM} / \mathrm{RS}$ & - & - & - & - & - & - & - & - & - & - & + & + & - & - & - & $\mathrm{a}, \mathrm{c}$ \\
\hline \#Castelnavia fluitans Tul. \& Wedd. & $\mathrm{EM} / \mathrm{RS}$ & - & - & - & - & - & - & + & + & - & - & - & - & + & + & - & $\mathrm{a}, \mathrm{c}$ \\
\hline "Castelnavia monandra Tul. \& Wedd. & $\mathrm{EM} / \mathrm{RS}$ & - & - & - & - & - & - & + & + & - & - & - & - & - & + & + & $\mathrm{a}, \mathrm{c}$ \\
\hline Castelnavia multipartita Tul. \& Wedd. & $\mathrm{EM} / \mathrm{RS}$ & - & - & - & - & - & - & + & + & - & - & - & - & - & - & + & $\mathrm{a}, \mathrm{c}$ \\
\hline${ }^{\#}$ Castelnavia noveloi C. Philbrick \& C. P. Bove & $\mathrm{EM} / \mathrm{RS}$ & - & - & - & - & - & - & - & - & - & - & - & - & + & + & + & $\mathrm{a}, \mathrm{c}$ \\
\hline $\begin{array}{l}\text { \#Castelnavia pendulosa (C. T. Philbrick \& C. P. Bove) C. Philbrick } \\
\text { \& C. P. Bove }\end{array}$ & $\mathrm{EM} / \mathrm{RS}$ & - & - & - & - & - & - & + & - & - & - & - & - & - & - & - & $\mathrm{a}, \mathrm{c}$ \\
\hline "Castelnavia princeps Tul. \& Wedd. & $\mathrm{EM} / \mathrm{RS}$ & - & - & - & - & - & - & + & + & - & - & - & - & - & + & + & $a, c$ \\
\hline \#Ceratolacis erytrolichen (Tul. \& Wedd.) Wedd. & $\mathrm{EM} / \mathrm{RS}$ & - & - & - & - & - & - & - & - & - & - & - & - & + & - & + & $a, c$ \\
\hline \#Jenmaniella ceratophylla Engl. & $\mathrm{EM} / \mathrm{RS}$ & - & - & - & - & - & + & + & + & - & - & - & - & - & - & - & $a, c$ \\
\hline \#Jenmaniella fimbriata P. Royen & $\mathrm{EM} / \mathrm{RS}$ & - & - & - & - & - & + & + & + & - & - & - & - & + & + & + & $\mathrm{a}, \mathrm{c}$ \\
\hline \#Jenmaniella tridactylitifolia (Engler) v. Royen & $\mathrm{EM} / \mathrm{RS}$ & - & - & - & - & - & - & + & + & - & - & - & - & - & - & - & $\mathrm{a}, \mathrm{c}$ \\
\hline \#Marathrum capillaceum (Pulle) v. Roeyn & $\mathrm{EM} / \mathrm{RS}$ & - & - & - & + & + & - & - & - & - & - & - & - & - & - & - & $\mathrm{a}, \mathrm{c}$ \\
\hline \#Marathrum foeniculaceum Bonpl. & $\mathrm{EM} / \mathrm{RS}$ & - & - & - & - & + & - & - & - & - & - & - & - & - & - & - & $\mathrm{a}, \mathrm{c}$ \\
\hline $\begin{array}{l}\text { \#Marathrum squamosum Wedd. var. phellandrifollium (Engler) P. } \\
\text { Royen }\end{array}$ & $\mathrm{EM} / \mathrm{RS}$ & - & - & - & - & + & + & - & - & - & - & - & - & - & - & - & $\mathrm{a}, \mathrm{c}$ \\
\hline \#Marathrum squamosum Wedd. var. squamosum & $\mathrm{EM} / \mathrm{RS}$ & - & - & - & - & + & + & - & - & - & - & - & - & - & - & - & $a, c$ \\
\hline \#Monostylis capillacea Tul. & $\mathrm{EM} / \mathrm{RS}$ & - & - & - & - & + & + & + & + & + & + & - & - & + & + & - & $a, c$ \\
\hline
\end{tabular}


Table 2. (Continuation)

\begin{tabular}{|c|c|c|c|c|c|c|c|c|c|c|c|c|c|c|c|c|c|}
\hline \multirow{2}{*}{ Family/ Species } & \multirow{2}{*}{ LF } & \multicolumn{2}{|c|}{$\mathrm{AC}$} & \multicolumn{2}{|c|}{ AP } & \multicolumn{2}{|c|}{ AM } & \multicolumn{2}{|c|}{ PA } & \multicolumn{2}{|c|}{ R0 } & \multicolumn{2}{|c|}{$\mathrm{RR}$} & \multicolumn{2}{|c|}{ TO } & \multirow{2}{*}{ END } & \multirow{2}{*}{$\mathrm{HAB}$} \\
\hline & & $\mathrm{F}$ & $S$ & $\mathrm{~F}$ & $S$ & $\mathrm{~F}$ & $S$ & $\mathrm{~F}$ & $S$ & $\mathrm{~F}$ & $S$ & $\mathrm{~F}$ & $S$ & $\mathrm{~F}$ & $S$ & & \\
\hline "Mourera alcicornis (Tul. ) Wedd. & EM/RS & - & - & - & - & - & - & + & + & - & - & - & - & - & - & + & $\mathrm{a}, \mathrm{c}$ \\
\hline \#Mourera elegans (Tul.) Baill. & EM/RS & - & - & - & - & - & - & + & + & - & - & - & - & - & - & + & $a, c$ \\
\hline \#Mourera fluviatilis Aublet & $\mathrm{EM} / \mathrm{RS}$ & - & - & + & + & - & + & + & + & - & - & + & + & - & - & - & $\mathrm{a}, \mathrm{c}$ \\
\hline \#Mourera weddelliana Tul. & $\mathrm{EM} / \mathrm{RS}$ & - & - & - & - & - & - & + & - & - & - & - & - & + & - & + & $\mathrm{a}, \mathrm{c}$ \\
\hline "Mourera monadelpha (Bong.) C.T.Philbrick \& C.P.Bove & $\mathrm{EM} / \mathrm{RS}$ & - & - & - & - & + & - & + & + & + & - & - & - & - & - & - & $\mathrm{a}, \mathrm{c}$ \\
\hline \#Oserya biceps Tul. \& Wedd. & $\mathrm{EM} / \mathrm{RS}$ & - & - & - & - & - & - & + & + & - & - & - & - & - & - & + & $a, c$ \\
\hline \#Oserya flabellifera Tul. \& Wedd & $\mathrm{EM} / \mathrm{RS}$ & - & - & - & - & - & - & - & - & - & - & - & - & + & - & + & $\mathrm{a}, \mathrm{c}$ \\
\hline \#Oserya perpusilla (Went) P. Royen & $\mathrm{EM} / \mathrm{RS}$ & - & - & - & - & + & + & - & - & - & - & + & + & - & - & - & $\mathrm{a}, \mathrm{c}$ \\
\hline \#Oserya sphaerocarpa Tul. \& Wedd. & $\mathrm{EM} / \mathrm{RS}$ & - & - & - & - & - & - & - & - & - & - & + & + & - & - & - & $a, c$ \\
\hline \#Podostemum flagelliforme (Tul. \& Wedd.) C. Philbrick \& Novelo & $\mathrm{EM} / \mathrm{RS}$ & - & - & - & - & - & - & - & - & - & - & - & - & + & + & - & $a, c$ \\
\hline${ }^{\#}$ Rhyncholacis carinata P. Royen & $\mathrm{EM} / \mathrm{RS}$ & - & - & + & - & - & + & - & - & - & - & - & - & - & - & + & $a, c$ \\
\hline${ }^{\#}$ Rhyncholacis crassipes Spruce & $\mathrm{EM} / \mathrm{RS}$ & - & - & - & - & + & + & - & - & - & - & - & - & - & - & + & $a, c$ \\
\hline${ }^{*}$ Rhyncholacis flagellifolia P. Royen & $\mathrm{EM} / \mathrm{RS}$ & - & - & + & - & + & + & - & - & - & - & + & + & - & - & + & $a, c$ \\
\hline "Rhyncholacis hydrocichorium Tul. & $\mathrm{EM} / \mathrm{RS}$ & - & - & - & - & + & + & + & + & - & - & - & - & - & - & - & $a, c$ \\
\hline "Rhyncholacis linearis Tul. & $\mathrm{EM} / \mathrm{RS}$ & - & - & - & - & - & + & + & - & - & - & - & - & - & - & + & $a, c$ \\
\hline \#Rhyncholacis minor P. Royen & $\mathrm{EM} / \mathrm{RS}$ & - & - & - & - & - & - & + & + & - & - & - & - & - & - & + & $a, c$ \\
\hline "Rhyncholacis nitelloides (Wedd.) P. Royen & $\mathrm{EM} / \mathrm{RS}$ & - & - & - & - & + & + & - & - & - & - & - & - & - & - & + & $\mathrm{a}, \mathrm{c}$ \\
\hline${ }^{*}$ Rhyncholacis oligandra Wedd. var. oligandra & $\mathrm{EM} / \mathrm{RS}$ & - & - & - & - & + & + & - & - & - & - & - & - & - & - & - & $a, c$ \\
\hline${ }^{\#}$ Rhyncholacis unguifera P. Royen & $\mathrm{EM} / \mathrm{RS}$ & - & - & - & - & + & + & - & - & - & - & - & - & - & - & + & $\mathrm{a}, \mathrm{c}$ \\
\hline "Rhyncholacis varians Spruce var. tricholoba Wedd. & $\mathrm{EM} / \mathrm{RS}$ & - & - & - & - & + & + & - & - & - & - & - & - & - & - & + & $a, c$ \\
\hline "Rhyncholacis varians Spruce var. varians & $\mathrm{EM} / \mathrm{RS}$ & - & - & - & - & + & + & - & - & - & - & - & - & - & - & + & $\mathrm{a}, \mathrm{c}$ \\
\hline \#Weddellina squamulosa Tul. var. squamulosa & $\mathrm{EM} / \mathrm{RS}$ & - & - & - & + & + & + & + & + & + & + & - & - & + & + & - & $a, c$ \\
\hline $\begin{array}{l}\text { \#Weddellina squamulosa Tul var. uaupensis (Benth. \& Hooker) } \\
\text { P. Royen }\end{array}$ & $\mathrm{EM} / \mathrm{RS}$ & - & - & - & - & + & + & - & - & - & - & - & - & - & - & + & $\mathrm{a}, \mathrm{c}$ \\
\hline \multicolumn{18}{|l|}{ Polygalaceae } \\
\hline *Asemeia monticola (Kunth) J.F.B.Pastore \& J.R.Abbott & A & - & + & + & + & + & + & + & + & - & - & + & + & - & - & - & $\mathrm{a}, \mathrm{c}$ \\
\hline *Polygala adenophora DC. & A & - & - & + & + & + & + & + & + & + & + & - & + & + & + & - & $c$ \\
\hline *Polygala appendiculata Vell. & A & - & - & - & - & + & - & + & + & - & - & + & - & - & - & + & $\mathrm{a}, \mathrm{c}$ \\
\hline *Polygala appressa Benth. & A & - & - & + & + & + & + & + & + & - & - & + & + & + & + & - & $a, c$ \\
\hline *Polygala hygrophila Kunth & A & + & + & + & + & + & + & + & + & - & - & + & + & + & + & + & $\mathrm{a}$ \\
\hline *Polygala longicaulis Kunth & A & - & - & + & + & + & + & + & + & - & - & + & + & + & + & + & $a, c$ \\
\hline *Polygala mollis Kunth & A & + & - & + & + & + & + & + & + & - & - & + & - & + & - & - & $a, c$ \\
\hline *Polygala paniculata L. & A & - & - & - & - & + & + & + & + & - & - & + & + & - & - & - & $\mathrm{a}$ \\
\hline *Polygala subtilis Kunth & A & - & - & - & - & + & + & + & + & - & - & + & + & - & + & - & $c$ \\
\hline *Polygala timoutou Aubl. & A & + & + & + & + & + & + & + & + & + & + & + & + & + & + & + & $a, c$ \\
\hline *Polygala trichosperma Jacq. & A & - & - & + & - & + & + & + & + & + & - & + & + & + & + & - & $c$ \\
\hline *Securidaca diversifolia (L.) S.F. Blake & A & - & - & + & + & + & + & + & + & + & + & + & + & + & + & - & $\mathrm{a}, \mathrm{c}$ \\
\hline *Securidaca lateralis A.W. Benn. & A & - & - & + & - & + & + & + & + & + & + & - & + & - & - & - & $\mathrm{a}, \mathrm{c}$ \\
\hline \#Securidaca prancei Wurdack & A & - & - & - & - & + & + & + & + & + & + & - & - & - & - & - & c \\
\hline \multicolumn{18}{|l|}{ Polygonaceae } \\
\hline Polygonum acuminatum Kunth & EM/A & + & + & + & + & + & + & + & + & + & + & + & + & + & + & - & $b, c$ \\
\hline Polygonum ferrugineum Wedd. & $\mathrm{EM} / \mathrm{A}$ & - & - & - & - & + & - & + & + & + & + & - & - & + & + & - & $a, b$ \\
\hline Polygonum punctatum Elliott & $E M / A$ & + & + & - & - & + & + & + & + & - & - & + & - & - & - & - & b \\
\hline \multicolumn{18}{|l|}{ Pontederiaceae } \\
\hline Eichhornia azurea (Sw.) Kunth & $\mathrm{EM} / \mathrm{FF}$ & - & - & + & + & + & + & + & + & + & + & + & + & + & + & - & $d$ \\
\hline Eichhornia crassipes (Mart.) Solms & $\mathrm{EM} / \mathrm{FF}$ & - & + & - & - & + & + & - & + & - & + & - & + & - & + & - & $d$ \\
\hline
\end{tabular}


Table 2. (Continuation)

\begin{tabular}{|c|c|c|c|c|c|c|c|c|c|c|c|c|c|c|c|c|c|}
\hline \multirow{2}{*}{ Family/ Species } & \multirow{2}{*}{ LF } & \multicolumn{2}{|c|}{$\mathrm{AC}$} & \multicolumn{2}{|c|}{ AP } & \multicolumn{2}{|c|}{ AM } & \multicolumn{2}{|c|}{ PA } & \multicolumn{2}{|c|}{ RO } & \multicolumn{2}{|c|}{$\mathrm{RR}$} & \multicolumn{2}{|c|}{ TO } & \multirow{2}{*}{ END } & \multirow{2}{*}{$\mathrm{HAB}$} \\
\hline & & $\mathrm{F}$ & S & $\mathrm{F}$ & S & $\mathrm{F}$ & $S$ & $\mathrm{~F}$ & $S$ & $\mathrm{~F}$ & $S$ & $\mathrm{~F}$ & $S$ & $\mathrm{~F}$ & S & & \\
\hline Eichhornia diversifolia (Vahl) Urb. & $\mathrm{EM} / \mathrm{FF}$ & + & + & - & - & - & + & + & + & - & + & - & + & + & + & - & $b, c$ \\
\hline Pontederia rotundifolia L. f. & EM & - & - & - & - & - & + & + & + & + & - & - & - & - & + & - & $a, c$ \\
\hline \multicolumn{18}{|l|}{ Pteridaceae } \\
\hline Ceratopteris pteridoides (Hook.) & $\mathrm{EM} / \mathrm{FF}$ & - & + & - & + & + & + & + & + & - & - & - & + & - & - & - & a,c \\
\hline \multicolumn{18}{|l|}{ Rubiaceae } \\
\hline *Alibertia latifolia (Benth.) K. Schum. & A & + & + & + & - & + & + & + & + & + & - & + & - & + & + & - & c \\
\hline \#*Borreria ocymoides (Burm.f.) DC. & A & - & - & - & - & + & + & - & + & - & - & - & + & - & + & - & c \\
\hline \# *Borreria verticillata (L.) G. Mey. & A & + & + & + & + & + & + & + & + & + & + & + & + & + & + & - & c \\
\hline \#*/sertia parviflora Vahl & A & - & - & - & - & + & + & + & + & - & - & + & + & - & - & - & c \\
\hline \#*Oldenlandia lancifolia (K. Schum.) DC. & A & + & + & - & - & + & + & + & + & + & + & - & + & - & + & - & c \\
\hline *Oldenlandia tenuis K. Schum. & A & - & - & - & - & + & + & + & + & - & + & + & - & - & - & - & c \\
\hline \# *Palicourea corymbifera (Müll. Arg.) Standl. & A & + & + & - & + & + & + & + & + & + & + & + & + & - & + & - & c \\
\hline *Psychotria poeppigiana Müll. Arg. & A & + & + & + & + & + & + & + & + & + & + & + & + & + & + & - & a,c \\
\hline *Rosenbergiodendron densiflorum (K.Schum.) Fagerl. & A & - & - & - & - & + & + & + & + & + & + & - & - & - & - & - & c \\
\hline *Sipanea pratensis Aubl. & A & - & - & + & + & + & + & - & + & - & + & + & + & - & - & + & a,c \\
\hline \multicolumn{18}{|l|}{ Salviniaceae } \\
\hline Azolla microphylla Kaulf. & FF & - & + & - & - & + & + & - & + & - & - & - & - & - & - & - & a,c \\
\hline Salvinia auriculata Aubl. & FF & - & - & + & + & + & + & + & + & - & - & + & + & + & + & - & $d$ \\
\hline \multicolumn{18}{|l|}{ Santalaceae } \\
\hline *Phoradendron platycaulon Eichler & A & - & - & - & - & + & + & + & + & + & + & - & + & - & - & - & c \\
\hline \multicolumn{18}{|l|}{ Sapindaceae } \\
\hline Cardiospermum halicacabum L. & A & + & + & + & + & + & + & + & + & - & - & + & + & - & - & - & $\mathrm{a}, \mathrm{c}$ \\
\hline \#Cupania cinerea Poepp. & A & + & + & + & + & + & + & + & + & + & + & + & + & - & - & - & c \\
\hline \#*Paullinia stipularis Benth. & A & - & - & - & - & + & + & + & + & + & - & - & - & - & - & + & $a, c$ \\
\hline Scrophulariaceae & & & & & & & & & & & & & & & & & \\
\hline \#Capraria biflora L. & A & - & - & - & - & + & + & - & - & - & - & - & - & - & - & - & c \\
\hline Solanaceae & & & & & & & & & & & & & & & & & \\
\hline *Physalis angulata L. & A & + & + & + & - & + & + & + & + & + & + & - & - & - & - & - & c \\
\hline *Solanum anceps Ruiz \& Pav. & A & + & + & + & - & + & + & - & - & + & + & - & - & - & - & - & $c$ \\
\hline *Solanum crinitum Lam. & A & + & + & + & + & + & + & + & + & + & + & + & + & + & + & - & c \\
\hline *Solanum jamaicense Mill. & A & + & + & + & + & + & + & + & + & + & + & - & - & - & - & - & c \\
\hline *Solanum sisymbriifolium Lam. & A & + & + & - & - & - & + & - & - & + & + & - & - & - & + & - & c \\
\hline *Solanum thelopodium Sendtn. & A & + & + & - & - & + & + & - & - & + & + & - & - & - & - & - & c \\
\hline Sphenocleaceae & & & & & & & & & & & & & & & & & \\
\hline Sphenoclea zeylanica Gaertn. & $\mathrm{EM} / \mathrm{A}$ & + & + & + & + & + & + & + & + & + & + & - & - & - & - & - & c \\
\hline Thelypteridaceae & & & & & & & & & & & & & & & & & \\
\hline " Cyclosorus interruptus (Willd.) H. Itô & EM & - & - & - & - & - & - & - & - & - & - & - & + & - & - & - & a,c \\
\hline *Thelypteris serrata (Cav.) Alston & EM & + & + & + & + & + & + & + & + & + & + & + & - & - & + & - & $\mathrm{a}, \mathrm{c}$ \\
\hline Typhaceae & & & & & & & & & & & & & & & & & \\
\hline Typha domingensis Pers. & EM & - & - & - & - & - & - & + & + & - & - & - & - & + & + & - & a,c \\
\hline Verbenaceae & & & & & & & & & & & & & & & & & \\
\hline *Lantana camara L. & A & + & + & + & + & + & + & - & + & + & + & + & + & + & + & - & c \\
\hline *Lantana canescens Kunth & A & - & - & - & - & + & + & - & + & - & - & - & - & - & + & - & c \\
\hline *Stachytarpheta angustifolia (Mill.) Vahl & A & - & - & + & + & - & + & + & + & - & - & - & + & - & - & - & c \\
\hline Vitaceae & & & & & & & & & & & & & & & & & \\
\hline *Cissus erosa Rich. & A & + & + & + & + & + & + & + & + & + & + & + & + & + & + & + & c \\
\hline
\end{tabular}


Table 2. (Continuation)

\begin{tabular}{|c|c|c|c|c|c|c|c|c|c|c|c|c|c|c|c|c|c|}
\hline \multirow{2}{*}{ Family/ Species } & \multirow{2}{*}{ LF } & \multicolumn{2}{|c|}{$\mathrm{AC}$} & \multicolumn{2}{|c|}{ AP } & \multicolumn{2}{|c|}{ AM } & \multicolumn{2}{|c|}{ PA } & \multicolumn{2}{|c|}{ RO } & \multicolumn{2}{|c|}{$\mathrm{RR}$} & \multicolumn{2}{|c|}{ TO } & \multirow{2}{*}{ END } & \multirow{2}{*}{$\mathrm{HAB}$} \\
\hline & & $\mathrm{F}$ & $S$ & $\mathrm{~F}$ & $S$ & $\mathrm{~F}$ & $S$ & $\mathrm{~F}$ & $S$ & $\mathrm{~F}$ & $S$ & $\mathrm{~F}$ & $S$ & $\mathrm{~F}$ & $S$ & & \\
\hline${ }^{\star}$ Cissus gongylodes (Baker) Planch. & A & + & + & - & - & - & - & + & + & - & + & - & - & - & + & - & c \\
\hline \multicolumn{18}{|l|}{ Xyridaceae } \\
\hline *Xyris jupicai Rich. & $\mathrm{EM} / \mathrm{A}$ & + & + & + & + & + & + & + & + & + & + & + & + & + & + & - & $\mathrm{a}, \mathrm{c}$ \\
\hline *Xyris macrocephala Vahl & $\mathrm{EM} / \mathrm{A}$ & + & + & + & + & + & + & + & + & + & + & + & + & + & + & - & $\mathrm{a}, \mathrm{c}$ \\
\hline${ }^{\star}$ Xyris paraensis Poepp. ex Kunth & $\mathrm{EM} / \mathrm{A}$ & - & - & - & - & + & + & + & + & - & - & + & + & - & - & - & $a, c$ \\
\hline *Xyris savanensis Miq. & $\mathrm{EM} / \mathrm{A}$ & + & + & + & + & + & + & + & + & + & + & + & + & + & + & - & $a, c$ \\
\hline
\end{tabular}

\section{DISCUSSION}

The Neotropics have the highest number of species (984) of aquatic plants of the world (Chambers et al. 2008). Regarding the high representation of aquatic plants of the region within the Neotropics and the strong endemism, Northern Brazil can be considered a priority area for conservation of the world aquatic vegetation.

Floristic studies on aquatic macrophytes have pointed that species richness is related to area, among other factors (Thomaz et al. 2003; Ferreira et al. 2011; Moura-Júnior et al. 2013). So, the high richness of aquatic macrophytes of Northern Brazil can be explained by the wetland size $\left(138.000 \mathrm{~km}^{2}\right)$.

In addition, there are evidences of the relation between increase in plant species richness with decrease of latitudinal gradient (Signor 1990; Cox and Moore 2010). This relationship is widely accepted, based on various theories: temporal, spatial, competitive, predictive, climate stability and biological production (Blackburn and Gaston 1996; Buzas et al. 2002; Raven et al. 2007). Therefore, the bioclimatic features related to the low latitude of Northern Brazil can also explain the high richness of aquatic macrophytes in this region.

It is known that sampling effort can influence results in assessment of richness and/or floristics. Therefore, the highest richness of aquatic macrophytes recorded in lentic habitats is because they have been more surveyed in Northern Brazil. According to Moura-Junior et al. (2011a), some of the hydrological characteristics of lentic environments (e.g. reduced turbidity and high transparency) favor the heterogeneity of aquatic plants, which may also explain the high richness of these environments for Northern Brazil. This fact shows the need for widening studies in lotic habitats, e.g. rivers and waterfalls. Moreover, the large and quite unaccessible wetlands of Araguaia, Guaporé and River Negro remain largely undersampled for aquatic macrophytes.

The states of AP, AM, RR and PA presented higher richness than large wetlands in Brazil, such as the Pantanal - 138,000 $\mathrm{km}^{2}$ (Pott and Pott 2000) and the Paraná river floodplain - 2,500 km² (Ferreira et al. 2011), what reinforces the importance of the Northern region of Brazil as a priority for conservation of diversity the world aquatic plants.
Poaceae and Cyperaceae are also among the richest families for other Brazilian wetlands, such as the Pantanal (Pott et al. 2011) and the floodplains of the upper Paraná river (Thomaz et al. 2003) and São Francisco river (Moura-Júnior et al. 2011a; Campelo et al. 2012). Souza and Lorenzi (2008) estimate $c a$. 1500 species of Poaceae and 700 Cyperaceae for Brazil, widely distributed over a range of aquatic and/or terrestrial habitats. It is believed that Cyperaceae and Poaceae adapt to ecotonal areas due to efficient vegetative propagation (rhizomes and/or stolons), what justifies the presence of $C$. haspan, C. luzulae and $E$. interstincta in all states and analyzed habitats. These are species of wide distribution (MOBOT 2011).

The species richness of Fabaceae in our study converged with the checklist of aquatic macrophytes of Northeast of Brazil (Moura-Júnior et al. 2013), wherein this family is one of the five richest. Morphological, ecophysiological and reproductive plasticity could explain the richness of Fabaceae in ecotonal areas (between terrestrial and aquatic zones), justifying the high number of legumes in Northern Brazil (Moura-Júnior et al. 2013). E.g. Mimosa spp. produce numerous long-living seeds. The richness of Malvaceae is because many of the listed species (e.g. Sida spp. and Urena lobata L.) are rather weedy and colonize gaps left after flood.

We call attention to the yet unreported high richness (55 species) of Podostemaceae in our study, considering the scarcity of this family in reports on aquatic macrophytes for tropical regions. Furthermore, 25 species are endemic to Brazil. We point out that all the cited species of this family were recorded in field expeditions and collected by two of the authors. We believe that the gap of records of Podostemaceae in studies on macrophytes of Northern Brazil may be related to lack of specialists and/or sampling difficulties regarding such rheophytes, which occur in habitats of very difficult and dangerous access, such as rapids and waterfalls.

It is noteworthy that Podostemum flagelliformis (Tul. \& Wedd.) C. Philbrick \& Novelo has been collected only once (1840s) in Brazil. This species occurs only attached to rocks in river-rapids. The only known location for the species (Tocantins River, near Porto Nacional) is now flooded by the reservoir of a hydroelectric dam in the Tocantins River, near the town of Lajeado. Thus, the unique appropriate habitat 
does not longer exist. We conducted field studies in the region over two seasons $(2005,2006)$ and this species was not reencountered. Therefore, it is listed as critically endangered (possibly extinct).

Climate is considered the main biogeographic predictor of flora and fauna, and the most determinant factors in characterization of the system are solar radiation, temperature, moisture and atmospheric pressure (Briggs 1995; Cox and Moore 2010). The latitudinal variation of these factors (mainly temperature) can cause differences in terrestrial and/or aquatic vegetation (Raven et al. 2007). In this context, floristic similarity of aquatic plants between the regions North and Northeast of Brazil, as well as their dissimilarity in relation to other Brazilian wetlands can be explained by the latitudinal variation of the climate system.

It is also known that micro-climatic differences within the region can influence sedimentological, hydrological and limnological characteristics of the hydrographic basins, changing patterns of geographic distribution of species and, consequently, the floristic composition of aquatic macrophytes (Murphy et al. 2003; Jacobs and Macisaac 2009). Therefore, the differences in richness and floristic composition of aquatic macrophytes among states of Northern Brazil can also be attributed to sedimentological, hydrological and limnological features of the micro-watersheds.

Sampling effort may have also influenced the floristic variation among states. This can be verified by results of floristic similarity, which showed similar patterns to the platforms, such as AM and PA (states with highest diversity of aquatic macrophytes), and the blurring of floristic similarity of TO (lowest richness) compared to other states of northern Brazil.

The assemblages of amphibious and/or emergent plants exhibit high species richness within the community of aquatic macrophytes of tropical regions (Moura-Júnior et al. 2013), regarding the wide range of adaptation and resilience of these plants under environmental pressures exerted by different types of ecosystems (lotic, lentic and transitional). We attribute the high participation of amphibious and emergent plants to their morphophysiology, which allow them to survive in the aquatic-terrestrial interface (Henry-Silva et al. 2010; Ribeiro et al. 2011; Moura-Júnior et al. 2011a). Other probable causes of the high number of amphibious and/or emergent aquatic macrophytes are the strategies of dispersal and seed dormancy of such plants and the shallow waters of the studied ecosystems in Northern Brazil.

The range of adaptation of amphibious and/or emergent species to ecotonal habitats allows that records may have arisen from research on both aquatic and non aquatic habitats (Moura-Júnior et al. 2011b), what can also partly explain the high species richness in Northern Brazil. Quite numerous plants are rather terrestrial elsewhere, generally considered ruderal, e.g. some Amaranthaceae, Fabaceae, Poaceae, Solanaceae and Malvaceae, whereas they are able to grow in running water along flooded riversides and sediments. Within this perspective, the taxonomic records of assemblages strictly related to aquatic habitats (floating and submerged) are considered little representative in studies focused on communities of aquatic macrophytes, what we corroborated. Nevertheless, the high number of aquatic species recorded in our study evidenced that Northern Brazil can be considered rich in life forms.

SpeciesLink is a repository updated by Brazilian herbaria. So, some negative biases could happen due to delayed update of herbarium databanks, and underestimate richness. In counterpart, Flora do Brasil is a list of species revised by specialists, hence, it is considered a reliable database for inventories of plant species in Brazil. Nevertheless, in our study nine species recorded in the field and confirmed by specialists were not listed in Flora do Brasil. This suggests that the data of Flora do Brasil may also be underestimated. Even so, we suggest that in studies of richness or diversity the Flora do Brasil platform can be priorized, while the choice of platform is not important for floristic composition.

\section{CONCLUSIONS}

There is a rich and diversified flora of aquatic plants in Northern Brazil, evidenced by the number of recorded taxa and variety of life forms. We highlight the unreported high number of species (48) of Podostemaceae, of which 25 are endemic. The contrasting results obtained from the platforms SpeciesLink and Flora do Brasil for richness may partially arise from delay in updating herbaria data or finding of new records of species. In this context, we suggest to amplify floristic and taxonomic efforts in Northern Brazil, particularly in AC, $\mathrm{RO}$ and $\mathrm{TO}$, short of information upon aquatic plants. In the future, probably more aquatic species shall be added to this first checklist.

\section{ACKNOWLEDGEMENTS}

To Coordenaçáo de Aperfeiçoamento de Pessoal de Nível Superior (CAPES, Ministry of Education, Brazil) for a Visiting Professor grant to A. Pott and a post-doc grant to F. A. Ferreira. To the researchers Liliane Lima and Tassia Pinheiro, from Universidade Federal Rural de Pernambuco.

\section{REFERENCES}

Albuquerque, B.W.P. 1981. Plantas forrageiras da Amazônia Aquáticas Flutuantes Livres. Acta Amazonica, 11: 457:471.

ANA. 2012. Agência Nacional de Águas, (www.ana.gov.br). Accessed on $20 / 06 / 2012$.

APG III 2009. Angiosperm Phylogeny Group. An update of the Angiosperm Phylogeny Group classification for the orders and 
families of flowering plants: APG III. Botanical Journal of the Linnean Society, 161: 105-121.

Bianchini Jr., I.; Cunha-Santino, M.B.; Fushita, A.T.; Almeida, D.A.A.; Maia, A.T. 2010. Monitoramento das Macrófitas Aquáticas do Reservatório da Usina Hidrelétrica Luís Eduardo Magalhães (Estado de Tocantins, Brasil). Augmdomus, 2: 38-48.

Bicudo, C.E.M.; Tundisi, J.G.; Scheuenstuhl, M.C.B. 2010. Águas do Brasil: Análises estratégicas. Instituto de Botânica, São Paulo, 2010, 224p.

Blackburn, T.M; Gaston, K.J. 1996. A sideways look at patterns in species richness, or why there are so few species outside the tropics. Biodiversity Letters, 3: 44-53.

Briggs, J.C. 1995. Global Biogeography. Elsevier Science, Amsterdam, 1995, 452p.

Buzas, M.A.; Collins, L.S.; Culver, S.J. 2002. Latitudinal difference in biodiversity caused by higher tropical rate of increase. Proceedings of the National Academy of Sciences, 99: 7841-7843.

Buck, W.R.; Goffinet, B. 2000. Morphology and classification of mosses; In: Shaw J.A.; Goffinet, B. (ed.). Bryophyte Biology. Cambridge University Press, Cambridge, p.72-124.

Campelo, M.J.A.; Siqueira-Filho, J.A.; Cotarelli, V.M.; Souza, E.B.; Pimenta, W.A.; Pott, J.V. 2012 Macrófitas Aquáticas nas Áreas do Projeto da Integração do Rio São Francisco. In: Siqueira-Filho, J.A. (org.). Flora das Caatingas do Rio São Francisco. Andrea Jakobsson Estúdio Editorial, Rio de Janeiro, p.192-229.

Chambers, P.A.; Lacoul, P.; Murphy, K.J.; Thomaz, SM. 2008. Global diversity of aquatic macrophytes in freshwater. Hydrobiologia, 595: 9-26.

Clarke, K.R.; Gorley, R.N. 2006. PRIMER - Plymouth Routines in Multivariate Ecological Research. v.6. Primer-E, Plymout, 2006, 32p.

Costa-Neto, S.V.; Senna, C.D.F.; L.C.L.; Silva, S.R.M. 2007. Macrófitas aquáticas das Regiōes dos Lagos do Amapá, Brasil. Revista Brasileira de Biociências, 5: 618-620.

Costa-Neto, S.V.; Tostes, L.C.L.; Thomaz, D.O. 2003. Inventário Florístico das Ressacas das Bacias do Igarapé da Fortaleza e do Rio Curiaú. In: Takiyama, L.R.; Silva, A.Q. da (orgs.). Diagnóstico das Ressacas do Estado do Amapá: Bacias do Igarapé da Fortaleza e Rio Curiaú, Macapá-AP. CPAQ/IEPA and DGEO/SEMA, Macapá, p.1-22.

Cox, C.B.; Moore, P.D. 2010. Biogeography: An Ecological and Evolutionary Approach. $8^{\text {th }}$ ed. Blackwell, Oxford, 2010, 506p.

Cowell, R.K. 2013. EstimateS: Statistical estimation of species richness and shared species from samples. v. 9. persistent, URL<pulr.oclc.org/estimates $>$.

Esteves, F.A. 2011. Fundamentos de Limnologia. $3^{\text {rd }}$ ed. Editora Interciência, Rio de Janeiro, 2011, 826p.

Fassett, N.C. 1940. A Manual of Aquatic Plants. Univ. of Wisconsin Press, Wisconsin, 1940, 405p.

Ferreira, F.A.; Mormul, R.P.; Thomaz, S.M.; Pott, A.; Pott, V.J. 2011. Macrophytes in the upper Paraná river floodplain: checklist and comparison with other large South American wetlands. Revista de Biologia Tropical, 59: 541-556.
Filgueiras, T.S.; Nogueira, P.E.; Brochado, A.L.; Guala II, G.F. 1994. Caminhamento: um método expedito para levantamentos florísticos qualitativos. Cadernos de Geociências, 12: 39-43

Henry-Silva, G.G.; Moura, R.S.T.; Dantas, L.L.O. 2010. Richness and distribution of aquatic macrophytes in Brazilian semi-arid aquatic ecosystems. Acta Limnologica Brasiliensis, 22: 147-156.

IBGE. 2011. Instituto Brasileiro de Geografia e Estatística, (www. ibge.gov.br/). Accessed on 20/09/2011.

INMET. 2013. Instituto Nacional de Meteorologia, (http://www. inmet.gov.br/portal). Accessed on 30/10/2013.

Irgang, B.E.; Gastal-Jr., C.V.S. 1996. Macrófitas Aquáticas da Planície Costeira do RS. Editora da UFRGS, Porto Alegre, 1996, 290p.

Jacobs, M.J.; Macisaac, H.J. 2009. Modelling spread of the invasive macrophyte Cabomba caroliniana. Freshwater Biology, 54: 296-305.

Junk, W.J.; Piedade, M.T.F. 1993. Herbaceuos plants of the Amazon floodplain near Manaus: Species diversity and adaptations to the flood pulse. Amazoniana, 12: 467-484.

Junk, W.J.; Furch, K. 1980. Química da água e macrófitas aquáticas de rios e igarapés na Bacia Amazônica e nas áreas adjacentes. Acta Amazonica, 10: 611-633.

Lista de espécies da flora do Brasil. 2014. Jardim Botânico do Rio de Janeiro, (www.floradobrasil.jbrj.gov.br). Accessed on $15 / 09 / 2014$.

Lista de espécies da flora do Brasil. 2012. Jardim Botânico do Rio de Janeiro, (www.floradobrasil.jbrj.gov.br). Accessed on 30/10/2012.

Lolis, S.F.; Thomaz, S.M. 2011. Monitoramento da composição específica da comunidade de macrófitas aquáticas no reservatório Luis Eduardo Magalhães. Planta Daninha, 29: 247-258.

Magurran, A.E. 2004. Measuring biological diversity. Blackwell Publishing, Malden, 2004, 215p.

Manly, B.F.J. 1997. Randomization, Bootstrap and Monte Carlo Methods in Biology. $2^{\text {nd }}$ ed. Chapman and Hall, London. 1997, 300p.

Mateucci, S.D.; Colma, A. 1982. La Metodologia para el Estudio de la Vegetacion. Colección de Monografias Científicas - Série Biologia, 22: 1-168.

MOBOT. 2011. Missouri Botanical Garden, (www. missouribotanicalgarden.org). Accessed on 30/12/2011.

Moura-Júnior, E.G.; Lima, L.F.; Silva, S.S.L.; Paiva, R.M.S.; Ferreira, F.A.; Zickel, C.S.; Pott, A. 2013. Aquatic macrophytes of Northeastern Brazil: Checklist, richness, distribution and life forms. Check List, 9: 298-312.

Moura-Júnior, E.G.; Abreu, M.C.; Severi, W.; Lira, G.A.S.T. 2011 a. O gradiente rio-barragem do reservatório de Sobradinho afeta a composição florística, riqueza e formas biológicas das macrófitas aquáticas? Rodriguésia, 62: 731-742.

Moura-Júnior, E.G.; Lima, L.F.; Lima, P.B.; Almeida Jr., E.B.; Santos-Filho, F.S. 2011b. Análise crítica do conhecimento das macrófitas aquáticas do estado do Piauí In: Santos-Filho, F.S.; Soares, A.F.C.L. (orgs.). Biodiveridade do Piaui: Pesquisas e Perspctivas. CRV, Curitiba, p.173-190. 
Murphy, K.J.; Dickinson, G.; Thomaz, S.M.; Bini, L.M.; Dick, K.; Greaves, K. et al. 2003. Aquatic plant communities and predictors of diversity in a sub-tropical river floodplain: the upper Rio Paraná, Brazil. Aquatic Botany, 77: 257-276.

Oliveira, A.L.R.; Gil, A.S.B.; Bove, C.P. 2011. Hydrophytic Cyperaceae from the Araguaia river basin, Brazil. Rodriguésia, 62: 847-866.

Piedade, M.T.F.; Junk, W.; D’Ângelo, S.A. 2010. Aquatic herbaceous plants of the Amazon floodplains: state of the art and research needed. Acta Limnologica Brasiliensia, 22: 165-178.

Piedade, M.T.F.; Schoengart, J.; Junk, W.J. 2005. O manejo sustentável das áreas alagáveis da Amazônia Central e as comunidades de herbáceas aquáticas. Uakari, 1: 29-38.

Pinheiro, E.P.; Lolis, S.F. 2012. Influência da transparência da coluna de água na distribuição espaço-temporal de macrófitas aquáticas no reservatório Luis Eduardo Magalhães, rio Tocantins. Interface, 5: 66-75.

Pinheiro, M.N.M.; Hortêncio, M.M.; Evangelista, R.A.O. 2012. Distribuição espacial da biodiversidade de macrófitas aquáticas nos lagos da região Nordeste do estado de Roraima. Revista Geonorte, 1: 162- 174.

Pott, V.J.; Pott, A.; Lima, L.C.P.; Moreira, S.N.; Oliveira, A.K.M. 2011. Aquatic macrophyte diversity of the Pantanal wetland and upper basin. Brazilian Journal of Biology, 1: 255-263.

Pott, V.J.; Pott, A. 2000. Plantas aquáticas do Pantanal. Embrapa, Brasília, 2000, 414p.

Raven, P.; Evert, R.; Eichhorn, S. 2007. Biologia Vegetal. $7^{\text {nd }}$ ed. Editora Guanabara, Koogan, 2007, 738p.

Ratter, J.A.; Bridgewater, S.; Ribeiro, J.F. 2003. Analysis of floristic composition of the Brazilian cerrado vegetation III: comparison of the woody vegetation of 376 areas. Edinburgh Journal of Botany, 60: 57-109.

Ribeiro, J.P.N.; Takao, L.K.; Matsumoto, R.S.; Urbanetz, C.; Lima, M.I.S. 2011. Plantae, aquatic, amphibian and marginal species, Massaguaçu River Estuary, Caraguatatuba, São Paulo, Brazil. Check List, 7: 133-138.

Sanders, H.L. 1968. Marine benthic diversity: a comparative study. The American Naturalist, 102:243-282.
Simberloff, D.S. 1972. Properties of the rarefaction diversity measurement. The American Naturalist, 106:414-418.

Signor, P.W. 1990. The geological history of diversity. Annual Review of Ecology and Systematics, 21: 509-539.

Smith, A.R.; Pryer, K.M.; Schuettpelz, E.; Korall, P.; Schneider, H.; Wolf, P.G. 2006. A classification for extant ferns. Taxon, 55: 705-731.

Souza, L.S.; Nunes, R.O. 2011. Levantamento de macrófitas aquáticas no rio Méquens. Revista Facimed, 3: 211-223.

Souza, V.C.; Lorenzi, H. 2008. Botânica Sistemática: Guia ilustrado para identificação das famílias de Angiospermas da flora brasileira, baseado em APG II. $2^{\text {nd }}$ ed. Instituto Plantarum, São Paulo, 2008, 704p.

SPLink. 2014. Centro de Referência em Informação Ambiental, CRIA - Fundação de Amparo à Pesquisa do Estado de São Paulo, (www.splink.cria.org.br). Accessed on 15/09/2014.

SPLink. 2012. Centro de Referência em Informação Ambiental, CRIA - Fundação de Amparo à Pesquisa do Estado de São Paulo, (www.splink.cria.org.br). Accessed on 30/10/2012.

Tavares, A.S.; Odnetz, O.; Enricone, A. 2006. The Podostemaceae family in Amazonian rivers and insect community associated. Insula, 35:19-50.

Thomaz, S.M.; Souza, M.C.; Bini, L.M. 2003. Species richness and beta diversity of aquatic macrophytes in a large subtropical reservoir (Itaipu Reservoir, Brazil): the influence of limnology and morphometry. Hydrobiologia, 505:119-128.

Thornton, K.W.; Kimmel, B.L.; Payne, F.E. 1990. Reservoir limnology: Ecological per-spectives. John Wiley and Sons, Inc., New Jersey, 1990, 246p.

Trevelin, L.C.; Oliveira, F.; Souza, M.B.S.; Postali, T.C. 2007. Diversidade local de macrófitas aquáticas em águas brancas e pretas na Amazônia Central. In. Inpa (org). Ecologia da Floresta Amazônica - Curso de Campo. PBDFF - INPA, Manaus, p.1-6.

Tropicos. 2014. Missouri Botanical Garden (www.tropicos.org). Accessed on 30/04/2014.

Recebido em 11/06/2014

Aceito em 27/10/2014 